\title{
METAL-ENRICHED GASEOUS HALOS AROUND DISTANT RADIO GALAXIES: CLUES TO FEEDBACK IN GALAXY FORMATION ${ }^{1}$
}

\author{
Michiel Reuland, ${ }^{2,3,4}$ Wil van Breugel, ${ }^{2,5}$ Wim de Vries, ${ }^{2}$ Michael A. Dopita, ${ }^{6}$ Arjun Dey, ${ }^{7}$ \\ George Miley, ${ }^{4}$ Huub Röttgering, ${ }^{4}$ Bram Venemans, ${ }^{4}$ S. A. Stanford, ${ }^{2,3}$ Mark Lacy, ${ }^{8}$ \\ Hy Spinrad, 9 Steve Dawson, 9 Daniel Stern, ${ }^{10}$ and Andrew Bunker ${ }^{11}$ \\ Received 2006 July 24; accepted 2007 February 20
}

\begin{abstract}
We present the results of an optical and near-IR spectroscopic study of giant nebular emission-line halos associated with three $z>3$ radio galaxies, 4C 41.17, 4C 60.07, and B2 0902+34. Previous deep narrowband Ly $\alpha$ imaging revealed complex morphologies with sizes up to $100 \mathrm{kpc}$, possibly connected to outflows and AGN feedback from the central regions. The outer regions of these halos show quiet kinematics with typical velocity dispersions of a few hundred $\mathrm{km} \mathrm{s}^{-1}$ and velocity shears that can mostly be interpreted as being due to rotation. The inner regions show shocked cocoons of gas closely associated with the radio lobes. These display disturbed kinematics and have expansion velocities and/or velocity dispersions $>1000 \mathrm{~km} \mathrm{~s}^{-1}$. The core region is chemically evolved, and we also find spectroscopic evidence for the ejection of enriched material in 4C 41.17 up to a distance of $\approx 60 \mathrm{kpc}$ along the radio axis. The dynamical structures traced in the Ly $\alpha$ line are, in most cases, closely echoed in the carbon and oxygen lines. This shows that the Ly $\alpha$ line is produced in a highly clumped medium of small filling factor and can therefore be used as a tracer of the dynamics of high-redshift radio galaxies (HzRGs). We conclude that these HzRGs are undergoing a final jet-induced phase of star formation with ejection of most of their interstellar medium before becoming "red and dead" elliptical galaxies.
\end{abstract}

Key words: galaxies: active — galaxies: formation — galaxies: high-redshift galaxies: individual (B2 0902+34, 4C 60.07, 4C 41.17) - quasars: emission lines

\section{INTRODUCTION}

There is compelling evidence that in galaxies the formation and evolution of the central stellar bulge and the massive nuclear black hole are intimately related (Magorrian et al. 1998; Ferrarese \& Merritt 2000; Gebhardt et al. 2000). Understanding this coevolution of galaxy spheroids and their central massive black holes is one of the major outstanding issues in modern cosmology. Because they are readily located by their ultrasteepspectrum radio properties, the high-redshift radio galaxies (HzRGs; $z>3$ ) provide an efficient means to locate and study the environment and physics of newly forming galaxies. There are at least two reasons why HzRGs are key in attempts to understand the physical processes involved. First, they rank among the most luminous, largest, and most massive galaxies known in the early

\footnotetext{
${ }^{1}$ Based on observations at the W. M. Keck Observatory, which is operated as a scientific partnership among the University of California, the California Institute of Technology, and the National Aeronautics and Space Administration. The Observatory was made possible by the generous financial support of the W. M. Keck Foundation.

${ }^{2}$ Institute of Geophysics and Planetary Physics, Lawrence Livermore National Laboratory, Livermore, CA 94550, USA; wil@igpp.ucllnl.org.

3 Physics Department, University of California, Davis, CA 95616, USA.

${ }^{4}$ Sterrewacht Leiden, 2300 RA Leiden, the Netherlands.

${ }^{5}$ University of California, Merced, CA 95344, USA.

${ }^{6}$ Research School of Astronomy and Astrophysics, Australian National University, Weston Creek, ACT 2611, Australia.

${ }^{7}$ National Optical Astronomy Observatory, Tucson, AZ 85719, USA.

8 Spitzer Science Center, California Institute of Technology, Pasadena, CA 91125, USA.

9 Department of Astronomy, University of California, Berkeley, CA 94720, USA.

10 Jet Propulsion Laboratory, California Institute of Technology, Pasadena, CA 91109, USA.

${ }^{11}$ Institute of Astronomy, University of Cambridge, Cambridge CB3 OHA, UK.
}

universe (e.g., De Breuck et al. 2002). Second, we observe them early in the epoch of galaxy formation at a time when their supermassive black holes (SMBHs) are highly active and while their relativistic jets are interacting most strongly on their host galaxies.

Within the framework of the standard $\Lambda$ cold dark matter $(\Lambda \mathrm{CDM})$ scenario, it is believed that massive galaxies grow in a hierarchical fashion through the merging of smaller stellar and dark matter halo objects. Whether their central black holes grow in similar fashion or whether they are primordial objects (Loeb 1993; Silk \& Rees 1998; Kauffmann \& Haehnelt 2000), the energetic outflows and the ionizing radiation from these central active SMBHs are expected to profoundly influence the evolution both of their parent galaxies and of the surrounding environment. Recent models describing the formation of massive galaxies and clusters provide further evidence for the importance of this feedback (Benson et al. 2003; Springel et al. 2005). In addition, outflows could provide a source for the chemical enrichment of the intergalactic medium seen at high redshifts (e.g., Rauch et al. 2001; Aguirre et al. 2001). While there is consensus that feedback in some form must be important, there is an ongoing debate about which manifestations dominate and the scales on which they operate.

Because they are themselves massive galaxies in the early universe, HzRGs are located in the regions of large matter overdensities and within regions rich in forming galaxies. Indeed, many are known to be embedded in regions of dense interstellar medium (ISM) and in environments containing the earliest known galactic clusters (e.g., McCarthy 1993; van Ojik et al. 1996, 1997; Athreya et al. 1998; Pentericci et al. 2000; Papadopoulos et al. 2000). These gaseous reservoirs enable us to study in detail the feedback processes occurring at high redshift.

In an earlier paper (Reuland et al. 2003) we described the results from narrowband imaging observations of unprecedented 
sensitivity of three HzRGs (B2 0902+34, 4C 60.07, and 4C 41.17). These images were obtained at the Keck II $10 \mathrm{~m}$ telescope using custom-made, high-throughput interference filters with bandpasses centered at the redshifted Ly $\alpha$ line. The observations revealed very luminous $\left(L_{\mathrm{Ly} \alpha} \simeq 10^{45} \mathrm{ergs} \mathrm{s}^{-1}\right)$ and extended $(\approx 200 \mathrm{kpc})$ emission-line nebulae with spectacular features not previously seen, such as long filamentary structures, ionization cones, and multiple sharply bounded regions of enhanced emission, all indicative of strong interactions as expected from the scenario painted above. We argued that these extended Ly $\alpha$ nebulae might represent gas cooling in massive CDM halos, supplying new material for the continued growth of the galaxies at their center. This feeding could then be responsible for feedback mechanisms through radio jets, supernova explosions, and radiation pressure from the AGNs, resulting in large-scale outflows. The extended X-ray halo around $4 \mathrm{C} 41.17$ provides further evidence for a highly interactive environment in such systems (Scharf et al. 2003).

However, with only morphological data, important issues concerning possible origins for the filaments and large-scale structures well beyond the radio sources could not be tackled. Because Ly $\alpha$ is a resonance line, it is also important to resolve the question of whether the nebulae represent truly extended ionized halos or, rather, are due to Ly $\alpha$ photons which are produced near the central AGN and are scattered off neutral hydrogen halos. Many other questions concerning the structure, origin, and fate of the emission-line gas remain. For example, is the gas organized in shells, filaments, or cloudlets? What is the source of ionization, and what is the chemical composition of the gas? Can the outflows regulate the growth of the galactic bulge and black hole? Can they expel metals from the deep potential wells to enrich the intergalactic and intercluster medium?

In order to better understand the kinematics, abundances, and ionization mechanisms of these halos we obtained optical and near-IR spectra with the Keck telescopes to measure the extent, intensity, and kinematics of $\mathrm{Ly} \alpha$ and other nonresonance lines, [He II], [O II], and [O III], at various position angles across the nebulae. This paper discusses the results of these spectroscopic observations.

The structure of the paper is as follows: The sample selection, observations, and data analysis are described in $\S 2$. In $\S 3$ we present results for individual objects. Section 4 is a discussion of the observed kinematics and line ratios of the halos. The implications for the origin and fate of the emission-line halos are discussed in $\S 5$, and our conclusions are given in $\S 6$.

Here we adopt the concordance cosmological parameters $\Omega_{M}=0.27, \Omega_{\Lambda}=0.73$, and $H_{0}=71 \mathrm{~km} \mathrm{~s}^{-1} \mathrm{Mpc}^{-1}$. The age of the universe is $1.7-1.9 h_{71}^{-1} \mathrm{Gyr}$ at the redshifts $(z=3.4-3.8)$ of our galaxies, and the angular-to-linear transformation is $\approx 7.4 h_{71}^{-1} \mathrm{kpc} \operatorname{arcsec}^{-1}$.

\section{OBSERVATIONS AND DATA ANALYSIS}

\subsection{Sample Selection}

The three objects selected for the spectroscopic follow-up observations are shown in Table 1 with their positions and adopted redshifts. They were selected from among the galaxies observed in the course of our Keck imaging program (Reuland et al. 2003). The reasons for their inclusion in the spectroscopic program are summarized as follows.

We selected 4C 41.17 at $z=3.8$ because it was one of the first HzRGs to be discovered (Chambers et al. 1990) and for many purposes serves as an archetype HzRG. Optical (Dey et al. 1997)
TABLE 1

Radio Galaxy Sample

\begin{tabular}{|c|c|c|c|}
\hline Source & R.A. (J2000.0) & Decl. (J2000.0) & $z$ \\
\hline 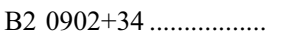 & 090530.11 & 340755.9 & 3.389 \\
\hline 4C $60.07 \ldots \ldots \ldots \ldots \ldots \ldots \ldots$ & 051255.17 & 603051.1 & 3.789 \\
\hline 4C $41.17 \ldots \ldots \ldots \ldots \ldots \ldots \ldots$ & 065052.14 & 413030.7 & 3.798 \\
\hline
\end{tabular}

Notes.-Positions of the radio core (Carilli et al. 1994, 1997; Carilli 1995) and the systemic redshifts based on the He II line are adopted as the frame of reference in this paper. Units of right ascension are hours, minutes, and seconds, and units of declination are degrees, arcminutes, and arcseconds.

and submillimeter-wavelength observations (Dunlop et al. 1994; Ivison et al. 2000) have shown that it is a massive forming galaxy with a star formation rate of up to several thousand $M_{\odot} \mathrm{yr}^{-1}$. Recently, very extended X-ray emission was found around 4C 41.17, which follows the Ly $\alpha$ morphology closely (Scharf et al. 2003).

We selected 4C 60.07 ( $z=3.8$; Chambers et al. 1996; Röttgering et al. 1997) because it shows both spatially and kinematically resolved CO emission (Papadopoulos et al. 2000; Greve et al. 2005). Interestingly, in Reuland et al. (2003) it was found that the Ly $\alpha$ halo has a very extended $\left(76 h_{71}^{-1} \mathrm{kpc}\right)$ filament which appears orthogonal to the major axis of the $\mathrm{CO}$ and dust emission.

B2 $0902+34(z=3.4$; Lilly 1988$)$ was selected because it is thought to be a protogalaxy, dominated by young stars (Eisenhardt \& Dickinson 1992). So far, it is one of only a handful of HzRGs for which neutral hydrogen has been detected in absorption against the radio continuum (Uson et al. 1991; Cody \& Braun 2003).

\subsection{Optical and Near-IR Spectroscopy}

Figure 1 shows the different slit positions used in the program overlaid on contour representations of the narrowband Ly $\alpha$ images of the galaxies. In contrast to most previous spectroscopic studies of HzRGs, most of the slits were not placed directly along the radio axis. The specific instrumental setups are given in Table 2 . The data reduction techniques are described below.

\subsubsection{Optical Observations: LRIS}

Most of the optical observations were carried out using the Low-Resolution Imaging Spectrometer (LRIS; Oke et al. 1995) at the Cassegrain focus on the $10 \mathrm{~m}$ Keck I telescope. The data were collected with various instrumental setups using both the long-slit mode and multislit masks designed to obtain spectra for $\approx 15$ targets in the field simultaneously (as part of a survey looking for associated galaxies in the protocluster; S. D. Croft et al. 2007, in preparation). The red-sensitive LRIS-R camera was employed. This uses a Tektronix $2048 \times 2048$ CCD detector with a pixel scale of $0.215^{\prime \prime} \mathrm{pixel}^{-1}$.

All of the spectroscopic reductions were performed using standard methods and the NOAO IRAF ${ }^{12}$ package (Tody 1993). Skylines were used to improve the first-order wavelength calibration based on arc spectra to better than $0.3 \AA \mathrm{rms}$. The instrumental resolution was measured from the unblended skylines. Flux calibrations were performed using observations of standard stars such as Feige 110 and Feige 34 (Massey et al. 1988). The extended emission of 4C 41.17 filled the narrow slits of the multiobject spectroscopic program, rendering accurate sky subtraction

\footnotetext{
12 IRAF is distributed by the National Optical Astronomy Observatory, which is operated by the Association of Universities for Research in Astronomy, Inc., under cooperative agreement with the National Science Foundation.
} 

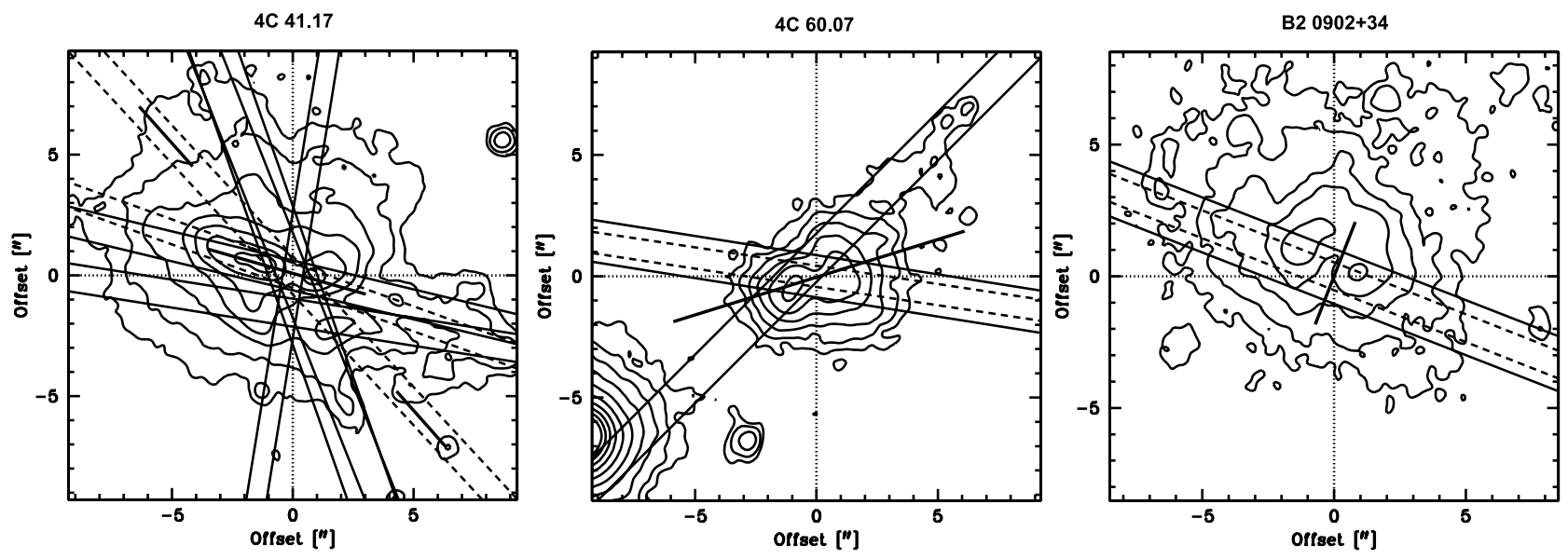

FIG. 1.-Contour representations of the Ly $\alpha$ emission-line halos around 4C 41.17 (left), 4C 60.07 (middle), and B2 $0902+34$ (right) with the various P.A.s at which they were studied overlaid. In this figure, north is at the top and east at the left. The solid and dashed lines correspond with slit positions of the optical and near-IR spectroscopy, respectively, and the dotted lines indicate the positions of the radio cores. The thick solid lines represent the direction of the radio axes. In the case of $4 \mathrm{C}$ 41.17 two axes are shown, corresponding to the inner and outer radio lobes. For each object the contours indicate observed surface brightnesses of $6.7 \times 10^{-19} \times$ $(6,12,25,50,100,200,400,800)$ ergs s$~^{-1} \mathrm{~cm}^{-2} \operatorname{arcsec}^{-2}$; see Reuland et al. (2003).

difficult. This does not seriously affect the kinematics and relative fluxes of interest for this paper.

\subsubsection{Optical Observations: ESI}

One set of observations along the filament of $4 \mathrm{C} 60.07$ was made during the night of UT 2001 February 25, using the Echelle Spectrograph and Imager (ESI; Sheinis et al. 2000) at the Cassegrain focus of the Keck II $10 \mathrm{~m}$ telescope in low-dispersion mode. The detector used is a high-resistivity MIT-Lincoln Labs $2048 \times 4096 \mathrm{CCD}$ with a plate scale of $0.154^{\prime \prime} \mathrm{pixel}^{-1}$. Exposures were broken into integrations of $1800 \mathrm{~s}$ each, one of which had to be halved because of time constraints. We performed $6^{\prime \prime}$ offsets between each integration. The data were rotated over an angle depending on the position of the object on the slit, in order to align the dispersion axes. The wavelength calibration varies slightly with slit position, hence we shifted the spectra along the dispersion axes. This first-order approximation is sufficiently accurate for the region of interest, $4300-9200 \AA$ (corresponding to approximately $900-1920 \AA \AA$ in the rest frame).
Subsequent data reduction was done using standard methods in IRAF.

\subsubsection{Near-IR Spectroscopy: NIRSPEC}

The near-IR spectra were obtained using the $10 \mathrm{~m}$ Keck II telescope with its Near-Infrared Spectrograph (NIRSPEC; McLean et al. 1998). The slit dimensions were $0.76^{\prime \prime} \times 42^{\prime \prime}$ slits giving low-resolution $(R \approx 1400-1900)$ spectra in wavelength ranges chosen to include the $\mathrm{H} \beta$, [O II], and [O III] lines of the target galaxies (see Table 2 for details). In this low-resolution mode, the $1024 \times 1024$ ALADDIN InSb detector has a plate scale of $0.143^{\prime \prime}$ pixel $^{-1}$. We obtained sets of $900 \mathrm{~s}$ integrations each with $\approx 5^{\prime \prime}-10^{\prime \prime}$ spatial offsets between exposures.

The NIRSPEC spectra need to be corrected for the spectral curvature and spatial distortions caused by the high-throughput optics. A general correction would require rectification onto a slit position-wavelength grid based on a wavelength solution from skylines and co-added exposures of a standard star. However, since no continuum is apparent in our spectra, we have only extracted

TABLE 2

Summary of Observations and Instrumental Setups Sorted by Position Angle

\begin{tabular}{|c|c|c|c|c|c|c|c|c|c|}
\hline Object & Date & Instrument & Setup & $\begin{array}{l}\text { Seeing } \\
(\operatorname{arcsec})\end{array}$ & $\begin{array}{c}\text { Slit } \\
(\operatorname{arcsec})\end{array}$ & $\begin{array}{l}\text { Res. } \\
(\AA)\end{array}$ & $\begin{array}{l}\lambda \text { Coverage } \\
(\AA)\end{array}$ & $\begin{array}{l}\text { P.A. } \\
\text { (deg) }\end{array}$ & $\begin{array}{l}\text { Obs. Time } \\
\text { (s) }\end{array}$ \\
\hline \multirow[t]{2}{*}{ B2 $0902+34 \ldots \ldots \ldots \ldots$} & 2002 Jan 16 & LRIS & LS $600 / 5000$ & 0.9 & 1.5 & 4 & $5150-7650$ & 68.7 & $2 \times 1800$ \\
\hline & 2002 Jan 7 & NIRSPEC & Low disp. N5 & 0.9 & 0.76 & 14 & $14600-17400$ & 68.5 & $6 \times 900$ \\
\hline \multirow[t]{3}{*}{ 4C $60.07 \ldots \ldots \ldots \ldots \ldots . .}$. & 2002 Jan 15 & LRIS & LS 600/7500 & 0.7 & 1.5 & 4 & $5450-7600$ & 81.0 & $2 \times 1800$ \\
\hline & 2001 Feb 25 & ESI & Low disp. & 0.8 & 1.0 & $13^{\mathrm{a}}$ & $4000-9600$ & 135.2 & $3.5 \times 1800$ \\
\hline & $2002 \operatorname{Jan} 7$ & NIRSPEC & Low disp. N6 & 0.9 & 0.76 & 14 & $15600-19800$ & 81.4 & $6 \times 900$ \\
\hline \multirow[t]{9}{*}{ 4C $41.17 \ldots \ldots \ldots \ldots \ldots . .}$. & 2001 Feb 24 & LRIS & LS 600/7500 & 0.7 & 1.5 & 6 & $5200-6100$ & 19.4 & $3 \times 1800$ \\
\hline & $2001 \mathrm{Feb} 23$ & LRIS & MOS 300/5000 & 0.6 & 1.0 & 4 & $4300-6600$ & 21.6 & $4 \times 1800$ \\
\hline & 1996 Dec 10 & LRIS & LS 400/8500 & 0.9 & 1.0 & 8 & $5500-9280$ & 76.5 & $28 \times 1200^{\mathrm{b}}$ \\
\hline & 2002 Jan 15 & LRIS & MOS 400/8500 & 0.7 & 1.0 & 6 & $5300-8000$ & 81.0 & $3 \times 1800$ \\
\hline & 1997 Feb 3 & LRIS & LS $600 / 5000$ & 0.9 & 1.0 & 5 & $4320-6850$ & 170.8 & $3 \times 1800$ \\
\hline & 2002 Jan 7 & NIRSPEC & Low disp. N6 & 0.9 & 0.76 & 14 & $15600-19800$ & 42.7 & $3 \times 900$ \\
\hline & 2002 Jan 7 & NIRSPEC & Low disp. N6 & 0.9 & 0.76 & 14 & $15600-19800$ & 70.2 & $5 \times 900$ \\
\hline & 2002 Jan 7 & NIRSPEC & Low disp. N7 & 0.9 & 0.76 & 14 & $20300-25000$ & 42.7 & $2 \times 900$ \\
\hline & $2002 \operatorname{Jan} 7$ & NIRSPEC & Low disp. N7 & 0.9 & 0.76 & 14 & $20300-25000$ & 67.8 & $4 \times 900$ \\
\hline
\end{tabular}

\footnotetext{
${ }^{\text {a }}$ Near the redshifted Ly $\alpha$ line at $\lambda=5825 \AA$; the resolution varies roughly linearly from $3 \AA$ at $3900 \AA$ to $40 \AA$ at $11000 \AA$.

b Spectropolarimetric observations, a detailed analysis of which was presented in Dey et al. (1997).
} 
small regions lying close to the emission lines. This approach requires only a simple rotation over an angle which depends on the wavelength of interest to provide a local calibration of the wavelength along the slit.

The data were flat-fielded and corrected for cosmic rays and bad pixels in the standard fashion. In order to remove the strong near-IR skylines, a sky frame scaled to the brightness of unsaturated skylines near the emission line of interest was subtracted. Subsequently, the frames were cropped, rotated, and co-added. Flux calibration was done with standard stars of spectral type A0 V, B3, and G4 and was consistent to within $10 \%$.

\subsection{Data Analysis}

For the data analysis the spectra were registered in position with radio maps from the literature (Carilli et al. 1994, 1997; Carilli 1995). The zero points of the spatial scales correspond with the radio core. This was achieved by identifying the core with the centroid of the continuum emission. If no continuum emission was visible, we identified the core with either the spatial region that showed the broadest line emission or by bootstrapping to spectra for which the core could be reliably identified. These results were then checked with the narrowband images, correlating with the peaks and dips in the observed intensity. This resulted in a total uncertainty of less than $0.5^{\prime \prime}$ in the relative spatial offsets.

The velocity scale used in the analysis is relative to the systemic velocity derived from the He II $\lambda 1640$ line (the adopted systemic redshifts are given in Table 1). The kinematic information was obtained from the two-dimensional spectra using a program written in IDL making use of the publicly available fitting routine MPFIT. ${ }^{13}$ The data were co-added within apertures matched to the seeing in order to increase the signal-to-noise ratio and ensure that the extracted spectra were not correlated. We then determined the velocity centroid and FWHM (corrected for instrumental broadening measured from unsaturated skylines and assuming that the widths add in quadrature). The peak and baseline fluxes were determined by fitting a single Gaussian and baseline to each trace. Single Gaussians provide a good fit to the outer regions, and, in order to allow a similarly direct comparison with the central regions, we have chosen to treat all regions consistently using the simpler approach. The single Gaussian decomposition for high surface brightness emission may break down when these regions are embedded in more smoothly varying, large-scale, low surface brightness envelopes.

\section{DYNAMICAL RESULTS}

Figures 2-4 show two-dimensional optical and near-IR spectra of 4C 41.17, 4C 60.07, and B2 0902+34 centered at the emission lines most relevant to our discussion. It is immediately obvious that many of the lines are very broad and show strong spatial variation in both their velocity centroids and their FWHMs. In some cases continuum emission was also detected, but only from the central regions, within $3^{\prime \prime}$ of the nucleus. Since the main interest of this paper is the extended emission, here we focus our discussion on the nebular lines.

For both 4C 41.17 and 4C 60.07 the oxygen-line profiles closely resemble the bright inner parts of the Ly $\alpha$ emission. Since self-absorption cannot be important in the strong forbidden lines, this suggests that the $\operatorname{Ly} \alpha$ line is giving useful information about the kinematic structure despite being subject to resonance scattering. This can only be possible if the interstellar gas is highly clumped. If this is true in the inner regions, it is probably also true in the outer regions. In any case, even if the line is being reso-

\footnotetext{
${ }^{13}$ Available at http://cow.physics.wisc.edu/ craigm/idl/fitting.html.
}

nantly scattered in the outer regions, it would likely still provide a fair (although possibly velocity-biased) measure of the neutral gas kinematics.

Figures 5-7 summarize radial velocities, FWHMs, and the run of relative surface brightness of the halos as a function of distance from the radio core for different position angles. These figures show clear evidence for a distinction between disturbed and more quiescent regions. The inner regions have higher surface brightnesses, are characterized by large velocity dispersions (FWHM $\approx$ $1500 \mathrm{~km} \mathrm{~s}^{-1}$ ), and seem to be embedded in low surface brightness regions with FWHMs of order $\approx 500 \mathrm{~km} \mathrm{~s}^{-1}$. In $\S 4$ we discuss the general implications of these diagrams. First, we describe the individual sources.

\subsection{Notes on Individual Objects}

\subsubsection{C 41.17: General Remarks}

Dey et al. (1997) discussed the brightest part $\left(2^{\prime \prime} \times 1^{\prime \prime}\right)$ of the extracted one-dimensional optical spectrum of $4 \mathrm{C} 41.17$ at P.A. $=76^{\circ}$ in detail. They determined a redshift $z=3.79786 \pm$ 0.00024 based on the He II line. Furthermore, they found evidence for stellar absorption lines and low polarization indicating that a young stellar population contributes significantly to the restframe UV continuum emission.

The two-dimensional spectra along the radio axis (P.A. $=$ $76^{\circ}$, plotted in Fig. 5) show that the Ly $\alpha$, [O II], and [O III] emission-line regions are very extended (over approximately $20^{\prime \prime}, 10^{\prime \prime}$, and $6^{\prime \prime}$, respectively). The Ly $\alpha$ and [O III] lines both show two separate components straddling the radio core with peak fluxes separated by about $3^{\prime \prime}$. Careful inspection reveals that these components are also present in the [O II] line, with the red part of the western component missing due to a skyline near $\lambda=17880 \AA$. The dip between the emission peaks is expected, since the narrowband $\operatorname{Ly} \alpha$ and radio image overlays show the core to be highly obscured (see Reuland et al. 2003).

The large extent of the nebula in the [O II] and [O III] emission lines immediately shows that the whole Ly $\alpha$ halo is rich in heavy elements. This immediately discounts the possibility that the emission-line halo represents infalling pristine gas. This point is discussed further below.

A second item of note is that the velocity gradient in the halo and the velocity dispersions observed in the lines are comparable. This shows that the halo is a highly turbulent and disordered structure.

The image overlays indicate that the position of the radio core is offset by $0.5^{\prime \prime}$ to the northeast from the central dip in the emission. In the spectra, the position of the radio core is revealed by a narrow high-velocity tail on the [O III] profile, extending out to $-1500 \mathrm{~km} \mathrm{~s}^{-1}$ and clearly visible in Figure 2 . In the Ly $\alpha$ profile, the nucleus is marked by a faint tail extending out to over $+2000 \mathrm{~km} \mathrm{~s}^{-1}$, the negative-velocity tail presumably being obscured by resonant scattering.

\subsubsection{C 41.17: Kinematics}

Dey et al. (1997) discerned the presence of both narrow and broad components in the central regions of the Ly $\alpha$ and $\mathrm{C}$ III] emission lines of $4 \mathrm{C} 41.17$ with FWHMs of $\approx 500-600$ and $\approx 1200-1300 \mathrm{~km} \mathrm{~s}^{-1}$, respectively. Figure 5 shows that these high velocity dispersions extend along the radio axis to the outer radio lobes. Beyond the limit of the radio lobes there is a break in velocity, the kinematics become more quiescent, and the velocity centroids change from being blueshifted $\left(-500\right.$ to $-700 \mathrm{~km} \mathrm{~s}^{-1}$ along the western lobe) to near systemic. Perpendicular to the radio axis (see Fig. 6) the kinematics follow a smooth gradient, 

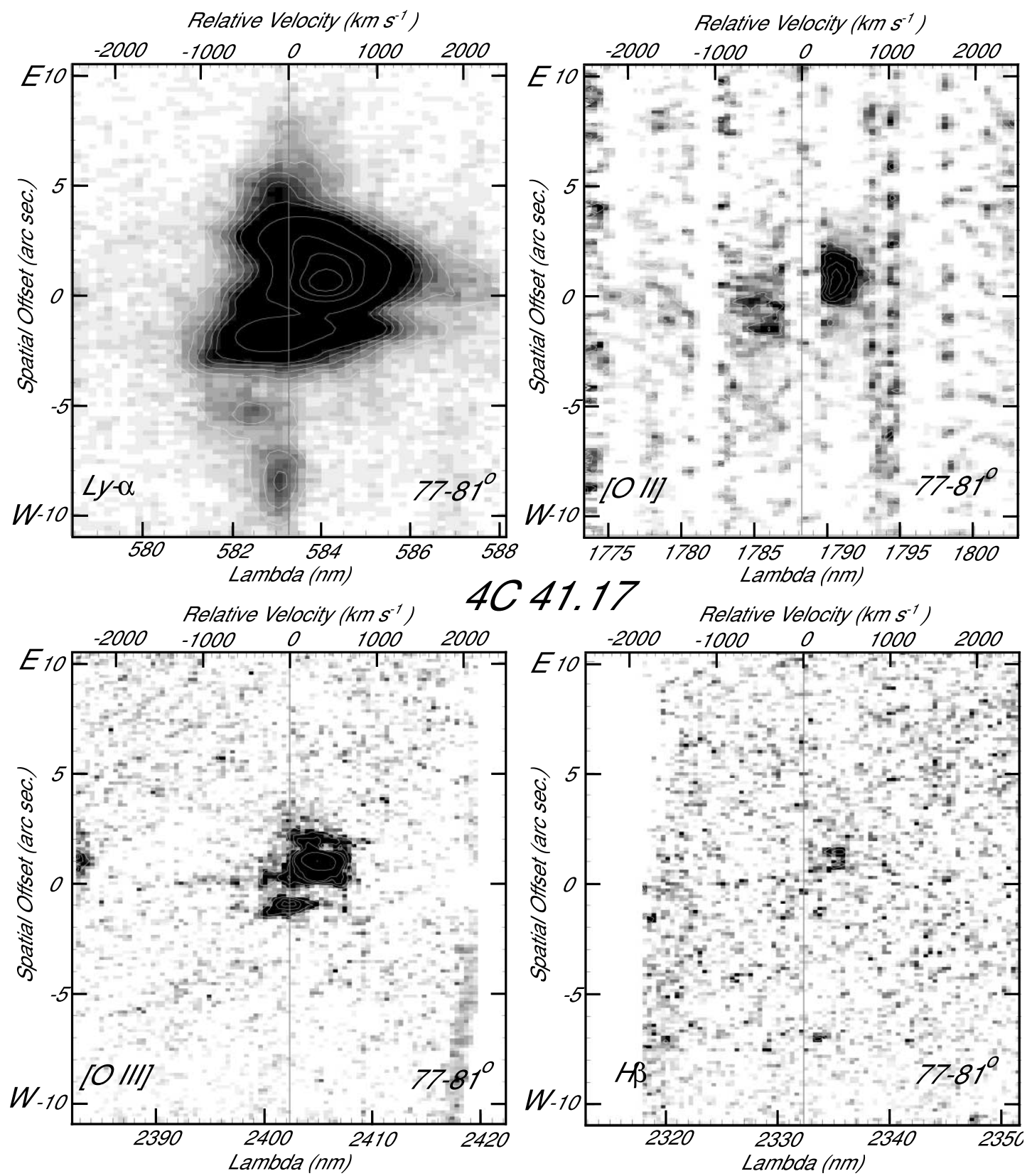

FIG. 2.-Gray-scale representations of the two-dimensional spectra of $4 \mathrm{C} 41.17$ centered at the Ly $\alpha$, [O II] $\lambda 3727, \mathrm{H} \beta$, and [O III] $\lambda 5007$ lines. The velocities are indicated relative to the systemic redshift determined from the He II line. The zero points of the spatial scales correspond with the position of the radio core as identified with the broad-line and continuum emission. Note that the peaks of the line emission corresponding with the position of radio knot B2 in Carilli et al. (1994) are $1^{\prime \prime}$ east of the nucleus. It can be seen (most easily in the $[\mathrm{O}$ III $] 25007$ spectrum) that there are $0.5^{\prime \prime}$ offsets between the broad lines and the central depression. The $[\mathrm{O}$ II] spectrum is rich in very strong skylines. These have been masked off to better show the structure of the $[\mathrm{O}$ II] line emission.

and the broad emission lines are found only in the central region of $\approx 3^{\prime \prime}(\approx 20 \mathrm{kpc})$ wide, identified by its sharp peak in peak surface brightness. This bright emission is associated with the inner radio lobes and has been successfully ascribed to radiative shocks at the boundary of the expanding cocoon (Bicknell et al. 2000).

Except for the high-velocity tail originating close to the AGN and discussed above, the oxygen lines show narrower line components than the Ly $\alpha$ line, with FWHMs of $\approx 600 \mathrm{~km} \mathrm{~s}^{-1}$. The measured velocities and velocity dispersions likely reflect the true motions of the gas, whereas $\operatorname{Ly} \alpha$ is additionally broadened by resonant scattering into the damping wings. The measurements on the [O III] line are likely to be much more reliable than those on the $[\mathrm{O} \mathrm{II}]$ line because the line is brighter, less reddened, and not so badly cut about by night-sky lines. Furthermore, corrections to the FWHM do not need to be made (in contradistinction to the $\left[\mathrm{O}_{\mathrm{II}}\right]$ doublet), and, finally, it can be observed closer to the nuclear region than [O $\mathrm{II}]$ because of its higher critical density (see, e.g., di Serego Alighieri et al. 1997).

Two very important results are found from the near-IR spectroscopy. First, as noted in the previous section, despite the obvious resonant broadening, the velocity structure of Ly $\alpha$ closely resembles that of [O III]. Second, there is [O II] emission in the velocity regime -200 to $-1000 \mathrm{~km} \mathrm{~s}^{-1}$ extending from the nucleus to $7^{\prime \prime}-8^{\prime \prime}$ west of the nucleus, whereas the $[\mathrm{O}$ III] emission appears to be much more centrally concentrated. We discuss this further in $\S 4.1$.

The Ly $\alpha$ emission appears to show a strong velocity shear in its kinematically quiescent outer parts as yet undisturbed by the 

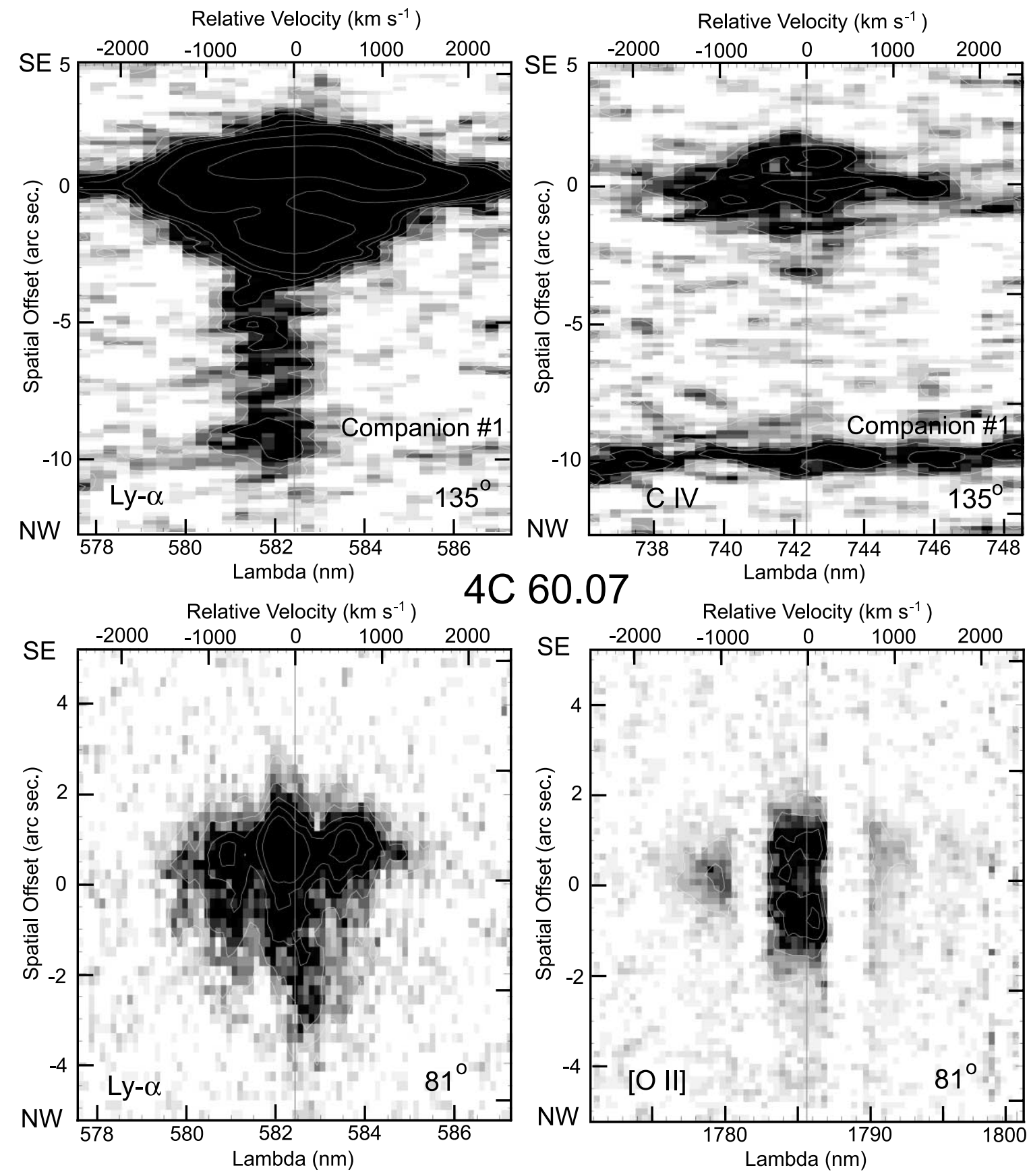

Fig. 3. - Similar to Fig. 2, but for 4C 60.07. Shown are the gray-scale representations of the two-dimensional spectra of the emission-line halo around $4 \mathrm{C} 60.07$. The top left and top right panels show Ly $\alpha$ and $\mathrm{C}$ IV emission, respectively, along the filament with P.A. $=135^{\circ}$. The bottom left and bottom right panels show Ly $\alpha$ and [O II] 23727 , respectively, along the radio axis with P.A. $=81^{\circ}$

expansion of the radio source along the radio axis. By contrast, the kinematic profiles obtained with slit positions perpendicular to the radio axis (see Fig. 6) show a fairly symmetric velocity distribution. This is indicative of an overall shear or rotation of the $\approx 200 \mathrm{kpc}$ diameter Ly $\alpha$ halo about its major axis.

The velocity structure of the bulk of the emission-line gas appears predominantly redshifted in the oxygen, $\mathrm{H} \beta$, and $\mathrm{Ly} \alpha$ lines. A similar redshift is seen in the extended $\mathrm{CO}(J=4-3)$ emission (De Breuck et al. 2005), suggesting that the AGN may be offset from the systemic redshift of the galaxy. The central CO component is situated at a relative velocity of $-125 \mathrm{~km} \mathrm{~s}^{-1}$. The location of this $\mathrm{CO}$ component coincides in spatial position and velocity with the Ly $\alpha$ gap between the "cloud" and the galaxy, as discussed in Reuland et al. (2003), suggesting that the dense molecular gas is absorbing the $\operatorname{Ly} \alpha$ emission at this location.

\subsubsection{C 60.07: General Remarks}

The ESI spectrum of $4 \mathrm{C} 60.07$ (P.A. $=135^{\circ}$; Fig. 3 ) shows a clear trace of continuum emission over approximately $3^{\prime \prime}$, which we associate with the position of the radio core and the unobscured optical nucleus. Comparison of this identification with the extent of the Ly $\alpha$ filament extending toward the northwest narrowband image (Fig. 1) and which is visible in the lower part of the spectrum shows that the nucleus has been located to within $0.5^{\prime \prime}$. Based on the He II line at $7855.7 \pm 1.1 \AA$ we infer a redshift $z=$ $3.7887 \pm 0.0007$, in agreement with Röttgering et al. (1997). 

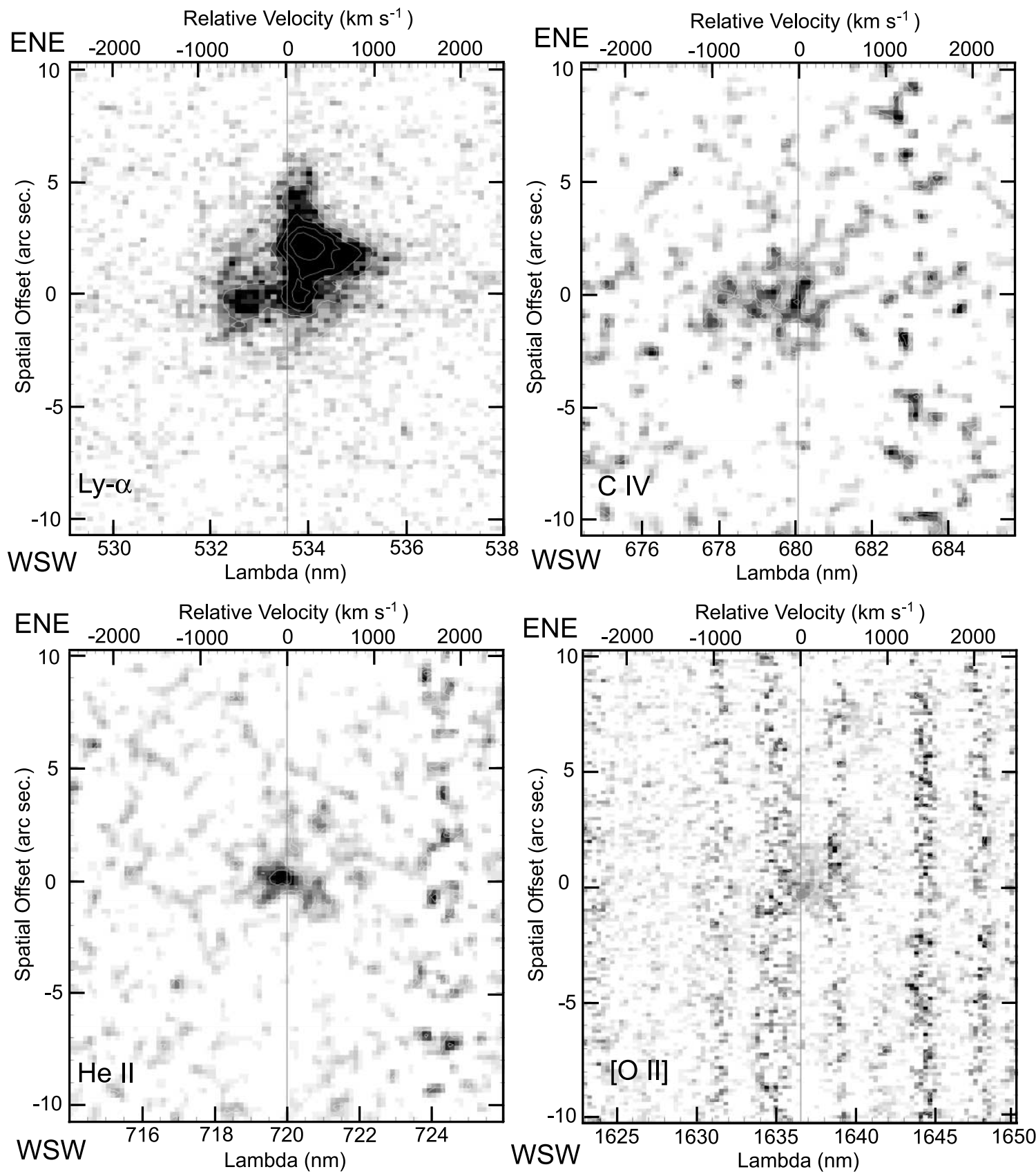

Fig. 4.-Similar to Fig. 2, but for B2 0902+34. Top left, line emission profile for Ly $\alpha$; top right, C IV; bottom left, He II; bottom right, [O п].

\subsubsection{C 60.07: Kinematics}

The two-dimensional Ly $\alpha$ emission line (Fig. 8) shows interesting structure: crescent-shaped clouds surround a gap at the position of the radio core near the systemic velocity of the galaxy. The spatial structure was expected from the narrowband image, but the kinematic substructure is unusual. A comparable lack of Ly $\alpha$ emission near the systemic velocity of the galaxy has been found in a Ly $\alpha$ galaxy associated with the SSA-22 protocluster (Wilman et al. 2005) and the large Ly $\alpha$ halo recently discovered by the Spitzer Space Telescope (Dey et al. 2005). It seems reasonable to attribute these velocity profiles to complex radiative transport effects of the resonantly scattered Ly $\alpha$ emission in dense (dusty) media (e.g., Neufeld 1990; Ahn 2004), since these sources are strong submillimeter (rest-frame far-IR) emitters (Papadopoulos et al. 2000; Chapman et al. 2001). However, the similarity of the profiles of the Ly $\alpha$ line and the $\mathrm{C}$ iv line in this region suggest, rather, an interaction of the radio lobe with a cocoon of material.

The morphologies of the Ly $\alpha$ and $\left[\mathrm{O}\right.$ II] spectra at P.A. $=81^{\circ}$ (Fig. 3) are remarkably similar, providing further justification for the use of Ly $\alpha$ as a useful tracer of the dynamics of these systems, despite the problems of resonance scattering. Both lines have very high velocity FWHMs of $\approx 100 \AA$ or $\approx 1600-1700 \mathrm{~km} \mathrm{~s}^{-1}$, rather large for HzRGs. They are, however, smaller than for the spectrum at P.A. $=135^{\circ}$, which shows a FWHM of $\approx 2600 \mathrm{~km} \mathrm{~s}^{-1}$. All of this is suggestive of the strong radiative shocks produced in an interaction of a powerful radio source with a surrounding dense ISM.

The emission-line profile across the Ly $\alpha$ gap can be fit with two Gaussian components with central wavelengths of $5839.9 \pm 0.5$ and $5806.8 \pm 0.4 \AA$ and FWHMs of $12.8 \pm 0.5$ and $10.6 \pm 0.3 \AA$, 

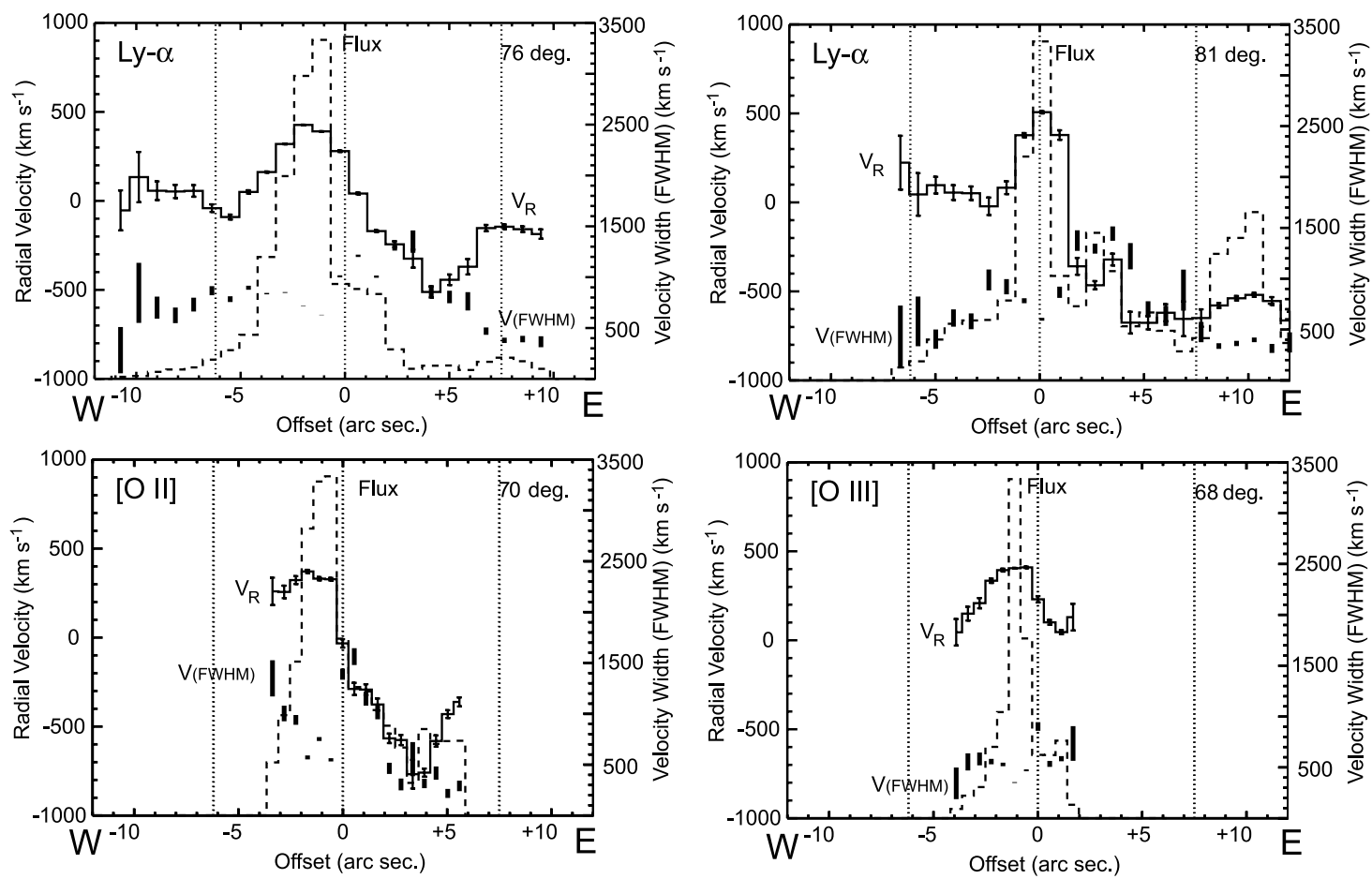

Fig. 5.-Relative velocities (solid lines), velocity dispersion (bars), and normalized surface brightness profiles (dashed lines) as determined from spectra with slit positions parallel to the radio axis for the $\mathrm{Ly} \alpha,[\mathrm{O} \mathrm{II}]$, and [O III] emission lines of $4 \mathrm{C} 41.17$. Top left, Ly $\alpha$ along P.A. $=76^{\circ}$; top right, Ly $\alpha$ for P.A. $=81^{\circ}$; bottom left, $\left[\mathrm{O}_{\mathrm{II}}\right]$ for P.A. $=70^{\circ}$; bottom right, $[\mathrm{O} \mathrm{III}]$ for P.A. $=68^{\circ}$. The spatial zero points correspond with the position of the radio core, and the dotted lines represent the position of the radio lobes projected on the slit. East is to the right. The bar and symbol sizes indicate the $1 \sigma$ uncertainties on the measurements. Note that the fits to the [O II] emission are affected by the strong skylines and that the slit along P.A. $=81^{\circ}$ does not go through the radio core.

respectively. However, it is probably better to fit the whole bright region to a single expanding shell by a velocity ellipsoid, estimated by fitting an ellipse to the position-velocity curve obtained for the two peaks in line intensity at each spatial position. The fit obtained depends somewhat on both the position angle of the observation and the line which is used to measure it, but all are consistent with an expansion velocity of $1100 \pm 200 \mathrm{~km} \mathrm{~s}^{-1}$.

\subsubsection{C 60.07: The Ly $\alpha$ Filament}

Perhaps the most striking feature of the emission-line nebula surrounding 4C 60.07 is the extended Ly $\alpha$ filament. Imaging showed this to be of fairly constant surface brightness, sharply bounded on the northeast side and much more "fluffy" and rather ill-defined on the southwest side close to the main emission region. The tip of the filament is cospatial with a small galaxy suggesting a causal connection. In Figure 9 we present the spectrum of this filament obtained with the ESI instrument.

Figures 8 and 10 show that the filament is offset from the systemic velocity of the radio source by approximately $-200 \mathrm{~km} \mathrm{~s}^{-1}$. The central wavelength fit by a single Gaussian yields $\lambda \approx$ $5818.1 \pm 0.3 \AA$ or $-175 \mathrm{~km} \mathrm{~s}^{-1}$ relative to the systemic velocity at $z=3.789$. The line has a FWHM of $20 \pm 1 \AA$ corresponding to a (deconvolved) velocity dispersion of $\approx 300 \mathrm{~km} \mathrm{~s}^{-1}$. The fact that there is no evidence for a strong $\left(>100 \mathrm{~km} \mathrm{~s}^{-1}\right)$ velocity gradient across the filament seems to indicate that it must be moving in the plane of the sky.

Observations with millimeter interferometers of the redshifted $\mathrm{CO}(J=4-3)$ transition in 4C 60.07 have found two kinematically and spatially separate gaseous reservoirs (Papadopoulos et al. 2000). This has recently been confirmed by VLA observations of the $\operatorname{CO}(J=1-0)$ transition (Greve et al. 2005). An overlay of the Ly $\alpha$ image with the millimeter observations (see
Fig. 5 in Reuland et al. 2003) suggests that the filament extends in a direction perpendicular to the major axis of the molecular gas, reminiscent of the (polar ejection) morphology of a galactic superwind. The $\mathrm{CO}(J=4-3)$ observations showed a gasrich narrow component with a FWHM of $\approx 150 \mathrm{~km} \mathrm{~s}^{-1}$ at a velocity of $-224 \mathrm{~km} \mathrm{~s}^{-1}$ to the galaxy. This same component has now been independently detected by its $\operatorname{CO}(J=1-0)$ emission at a relative velocity of $-220 \pm 40 \mathrm{~km} \mathrm{~s}^{-1}$ with a FWHM of $165 \mathrm{~km} \mathrm{~s}^{-1}$.

As is the case for $4 \mathrm{C} 41.17$, the optical spectroscopic observations show similar velocities for the molecular and emissionline gas. This corroborates the idea of a superwind, as it provides a dynamical connection between the filament and the gas fueling the nuclear AGN. If this is the case, the source of the ionization may be either shocks or photoionization from the central nucleus.

The superwind interpretation of the Ly $\alpha$ filament does, however, present certain difficulties. In particular, it is hard to understand how this feature, over $70 \mathrm{kpc}$ in length, manages to remain so well-collimated and kinematically quiescent over such a distance if it is related to a high-speed outflow (albeit in the plane of the sky). However, the filter cutoff may play an important role here, since the observed velocity of the filament is very close to it. Thus, it may give the appearance of a sharp boundary but may not be one in actuality. Possibly a slit placed over "empty sky" next to the filament would reveal other portions of the filament at different velocities.

An alternative possibility may be that it represents an accretion filament or a tidally stripped gas stream extending from the active galaxy. In this case both the quiescence of its velocity field and the similarity of the systemic velocities of the filament and the molecular reservoir would find a more natural explanation. 

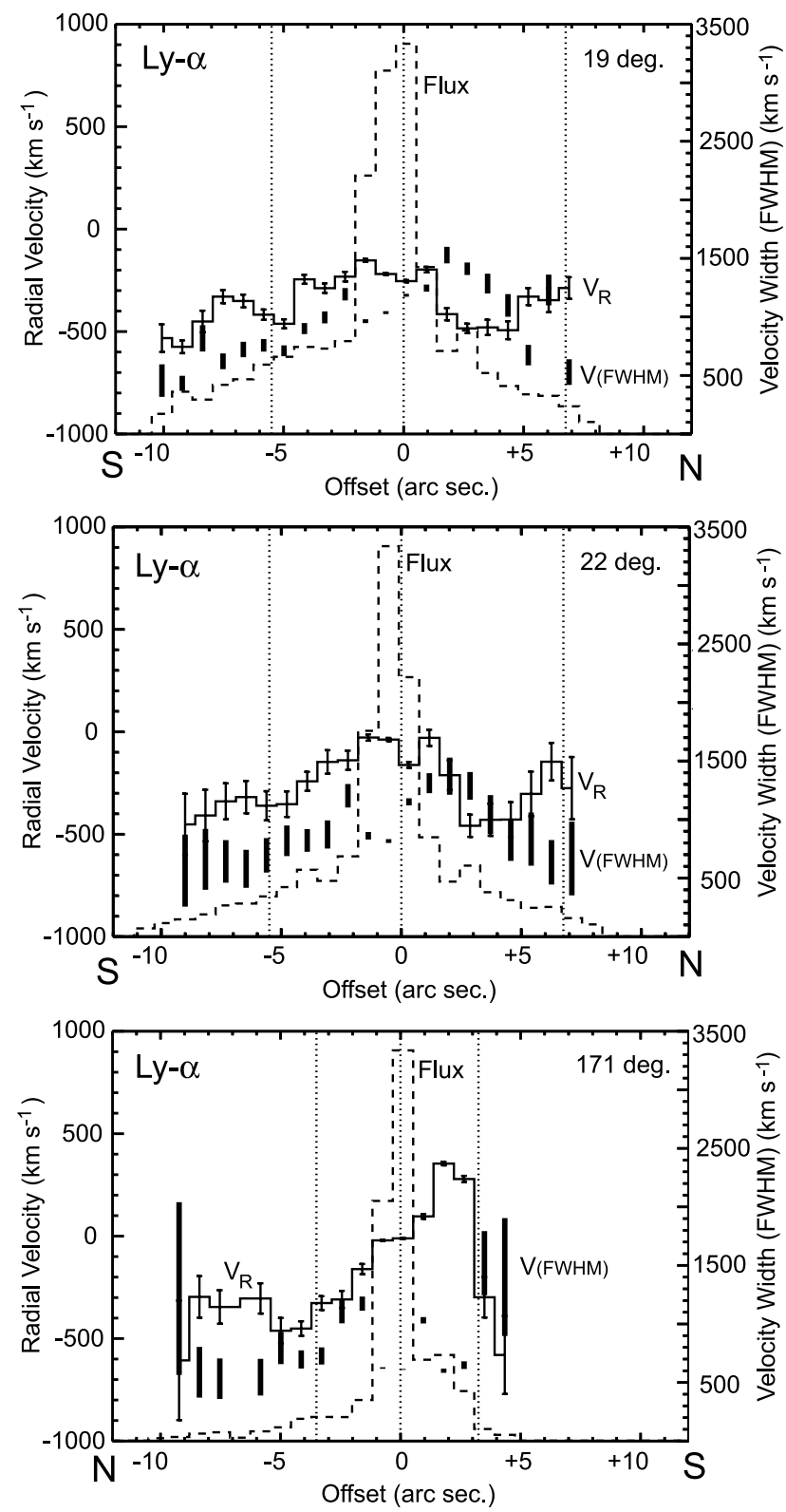

Fig. 6.- Similar to Fig. 5, but for the Ly $\alpha$ emission along the slits that lie more or less perpendicular to the radio axis of $4 \mathrm{C} 41.17$. Top, P.A. $=19^{\circ}$; middle, P.A. $=22^{\circ} ;$ bottom, P.A. $=171^{\circ}$.

Photoionization by the central AGN would then be the most probable cause of the ionization of the filament. On balance, this accretion or tidal-stripping scenario seems to provide the most convincing explanation for the filament.

The galaxy at the tip of the filament is most likely to be a chance superposition. Figure 9 shows the two-dimensional and one-dimensional spectra of this galaxy (companion 1) and a second companion galaxy (companion 2) which also fell on the slit. The spectrum of companion 1 is very similar to but fainter than that of companion 2 . The brighter galaxy shows an emission feature identified as the [O II] line and a break near $7000 \AA$, while the overall shape of the spectrum for the fainter galaxy is similar but without an evident break. Identifying the line at $7047.0 \AA$ with redshifted [O $\mathrm{II}] \lambda 3727$ yields a redshift of $z=$ 0.891 for the galaxy. This implies that it is a foreground object and not causally connected to the filament, despite its suggestive location.
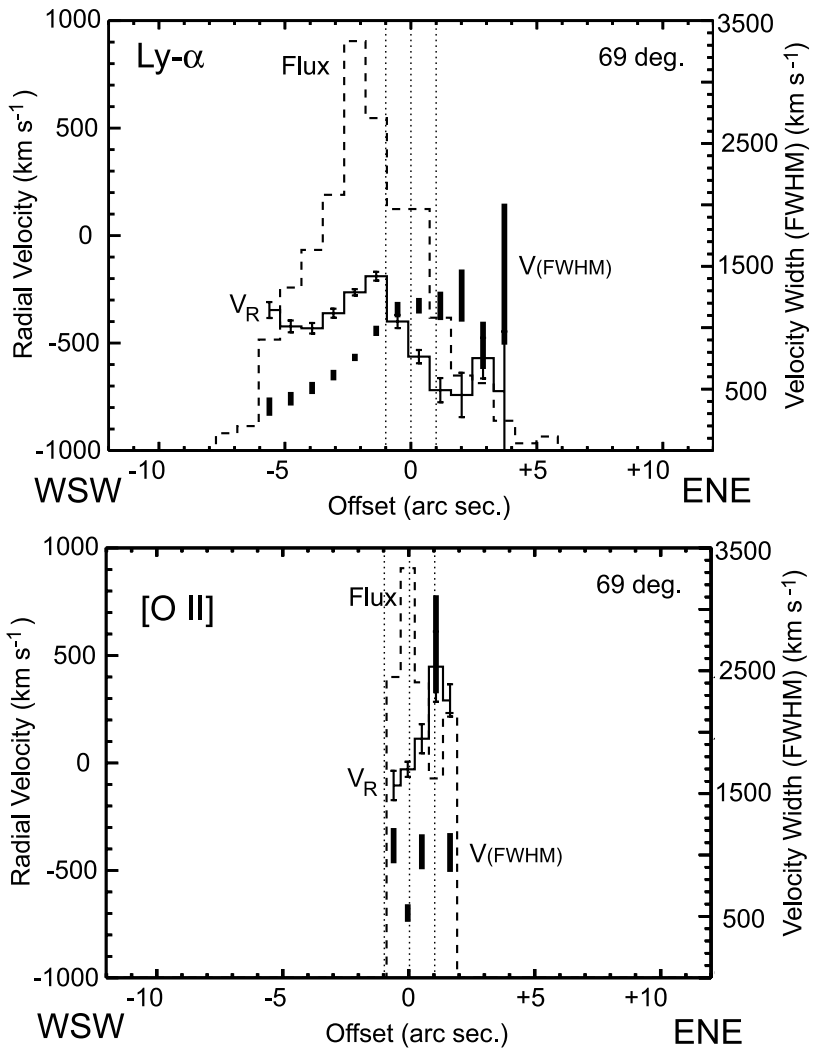

FIG. 7.-Similar to Fig. 5, but for B2 0902+34. Ly $\alpha$ (top) and [O II] (bottom) are both along P.A. $=69^{\circ}$.

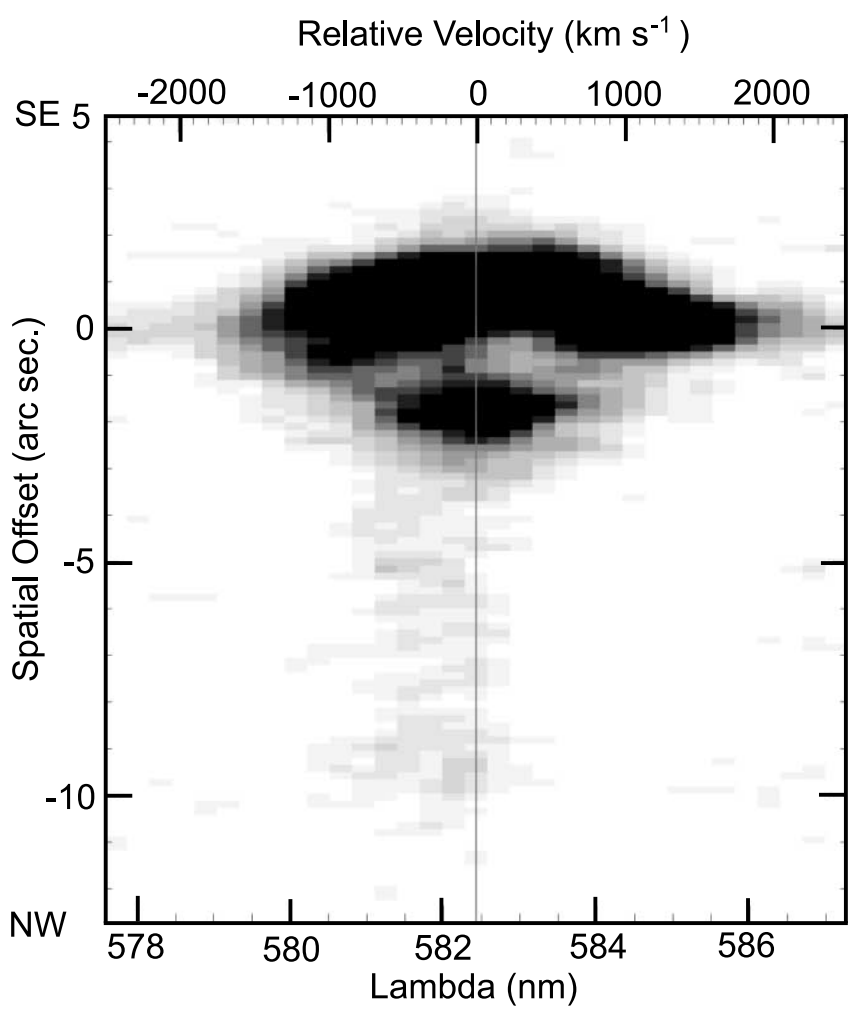

FIG. 8.-Expanded gray-scale representation of the two-dimensional spectrum of $4 \mathrm{C} 60.07$ centered at the redshifted Ly $\alpha$ line. Shown are the filament and the crescent-shaped arcs surrounding a depression in the emission that is identified with the location of both the radio core and the unobscured optical nucleus. This kinematical signature is probably the result of a shock interaction between the radio lobe and its cocoon. 

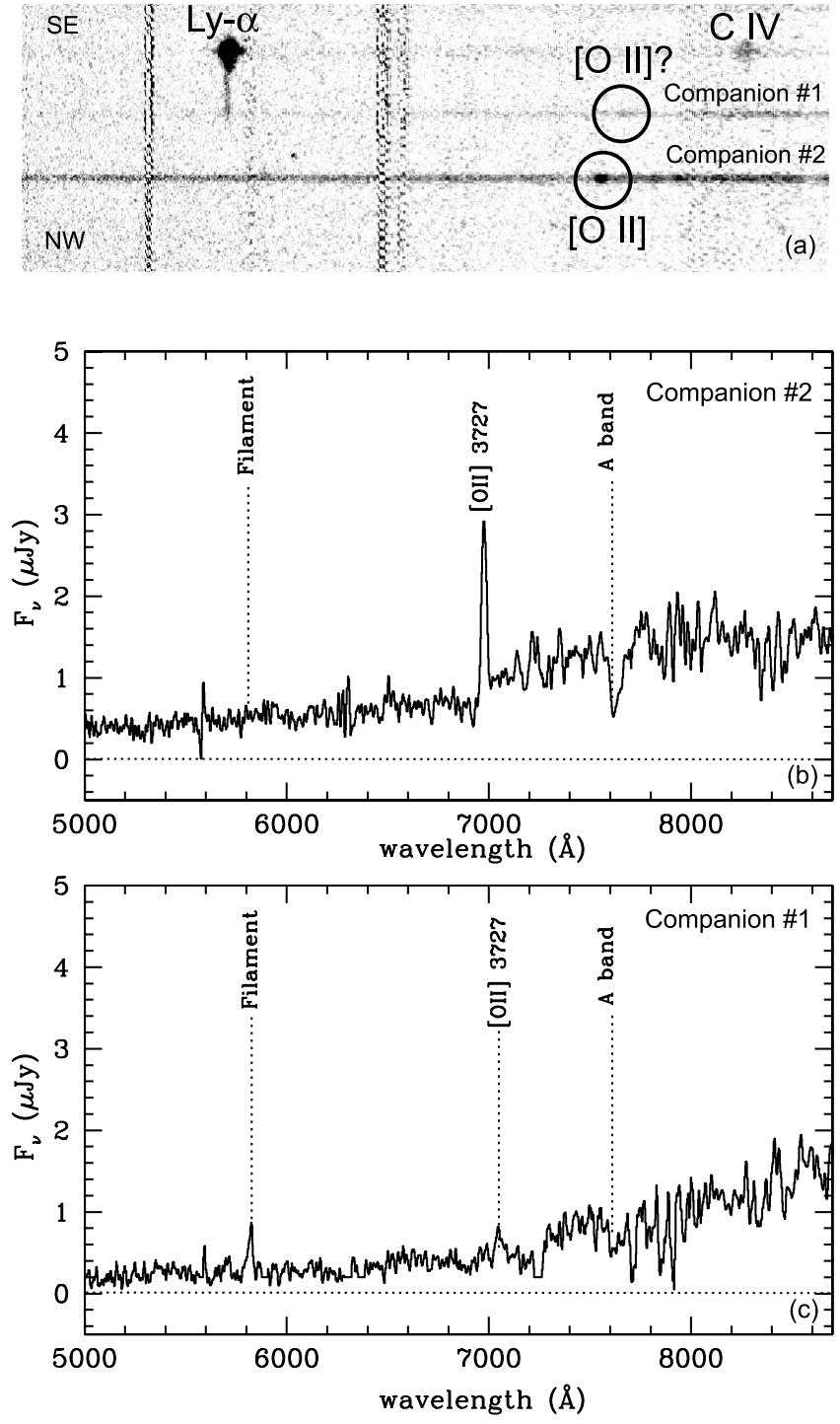

FIG. 9.- (a) Two-dimensional spectrum of the galaxy (companion 1) at the tip of the Ly $\alpha$ filament of $4 \mathrm{C} 60.07$, together with the spectrum of a brighter companion galaxy (companion 2) which happened to lie on the slit. The position of the galaxy might suggest a physical association with the Ly $\alpha$ filament of $4 \mathrm{C}$ 60.07. However, the spectrum of companion 1 bears a close resemblance to the brighter foreground galaxy companion 2 (shown in panels $c$ and $b$, respectively). The circles in $(a)$ indicate emission lines which are probably redshifted [O II] $\lambda 3727$ in both foreground galaxies, identified in the spectra of $(b)$ and $(c)$.

\subsubsection{B2 0902+34: General Remarks}

The optical spectrum obtained for B2 $0902+34$ is more sensitive than previous observations reported in the literature (Lilly 1988; Martin-Mirones et al. 1995). We have detected Ly $\alpha$ emission over an extent of $\approx 10^{\prime \prime}$ with a complex multicomponent spatial and velocity structure (see Fig. 4 ) and extended ( $\left.3^{\prime \prime}\right)$ continuum emission from the core over the entire wavelength range with an almost constant surface brightness. The Ly $\alpha$ profile shows galaxy-wide blueshifted absorption by neutral hydrogen.

As is evident from Table 3 and Figure 4, the inferred redshifts depend on both the emission line and the position of the aperture used. Much of this is due to the absorption optical depth effects in the line. The He II line at $7199.35 \pm 0.5 \AA$, extracted in a $2^{\prime \prime}$ wide aperture centered at the radio core, is probably most representative of the systemic velocity of the galaxy. From this we infer a redshift $z=3.3886 \pm 0.0003$. This is slightly less than that reported
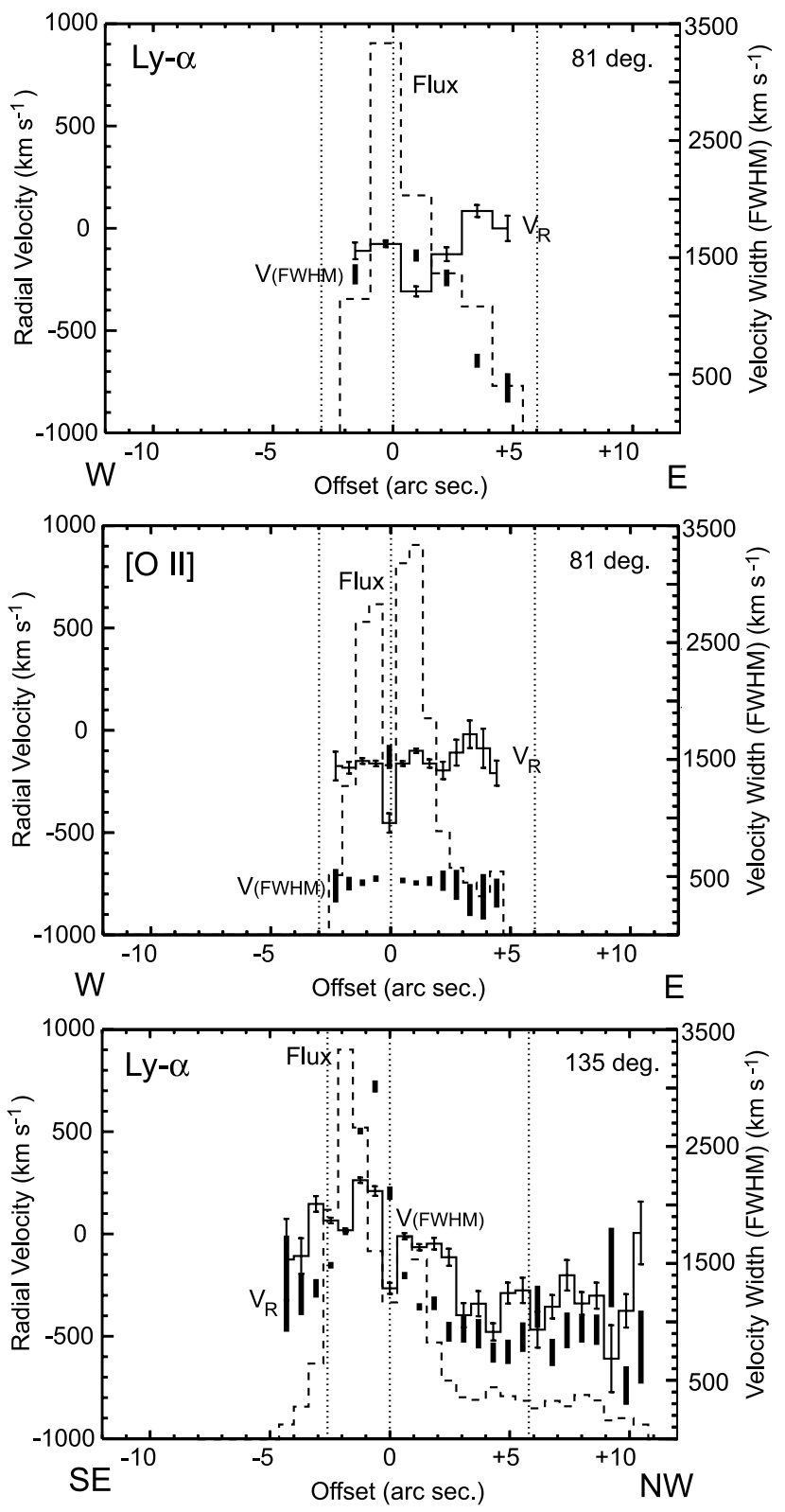

Fig. 10.- Similar to Fig. 5, but for 4C 60.07. Shown are Ly $\alpha$ (top) and [O II] (middle) emission along P.A. $=81^{\circ}$ and $\mathrm{Ly} \alpha$ emission (bottom) along P.A. $=135^{\circ}$.

previously by Lilly (1988) and Martin-Mirones et al. (1995). They inferred $z=3.395$ and 3.391, respectively. However, these reported redshifts are entirely consistent with redshift estimates we derive using other lines in the same aperture, emphasizing the difficulty of assigning single redshifts to such complex and selfabsorbed systems.

\subsubsection{B2 0902+34: Kinematics}

The He II line is only a few hundred $\mathrm{km} \mathrm{s}^{-1}$ wide and may be slightly extended, with its position of maximum surface brightness centered at the position of the radio nucleus. We can therefore argue that it arises from photoionization of the dense gas surrounding the nucleus. The [O II] line is broad and spatially extended, but it is too faint to allow a detailed comparison with the kinematics of the other lines.

Given that B2 $0902+34$ shows associated $\mathrm{H}$ I absorption against the radio continuum, it is important to see whether this is also present in the Ly $\alpha$ profile. This emission line shows a 
TABLE 3

Summary of Emission-Line Measurements

\begin{tabular}{|c|c|c|c|c|c|c|c|c|c|}
\hline Object & Line & $\begin{array}{l}\lambda_{\text {rest }} \\
(\AA)\end{array}$ & $\begin{array}{l}\lambda_{\text {obs }} \\
(\AA)\end{array}$ & $z$ & $\begin{array}{l}\text { FWHM } \\
(\AA)\end{array}$ & $\begin{array}{l}\text { FWHM } \\
\left(\mathrm{km} \mathrm{s}^{-1}\right)\end{array}$ & $\begin{array}{c}\text { Continuum Flux } \\
\left(10^{-17} \mathrm{ergs} \mathrm{s}^{-1} \mathrm{~cm}^{-2}\right)\end{array}$ & $\begin{array}{l}\text { Line Flux } \\
(\AA)\end{array}$ & $\mathrm{EW}_{\text {obs }}$ \\
\hline \multirow[t]{3}{*}{ B2 $0902+34$ (core) } & $\operatorname{Ly} \alpha$ & 1215.7 & $5334.69 \pm 0.09$ & $3.3883 \pm 0.0001$ & $21.9 \pm 0.2$ & $1233 \pm 13$ & $18.3 \pm 0.5$ & $7890 \pm 95$ & $431 \pm 12$ \\
\hline & C Iv (doublet) & 1549.0 & $6795.00 \pm 0.62$ & $3.3867 \pm 0.0004$ & $22.7 \pm 1.5$ & $1003 \pm 65$ & $16.3 \pm 0.4$ & $1770 \pm 70$ & $109 \pm 5$ \\
\hline & $\mathrm{He}$ II & 1640.5 & $7199.37 \pm 0.37$ & $3.3886 \pm 0.0002$ & $17.2 \pm 0.9$ & $718 \pm 37$ & $11.8 \pm 0.4$ & $1502 \pm 60$ & $127 \pm 7$ \\
\hline \multirow[t]{3}{*}{ B2 $0902+34$ (total) } & $\operatorname{Ly} \alpha$ & 1215.7 & $5336.26 \pm 0.23$ & $3.3896 \pm 0.0002$ & $21.4 \pm 0.4$ & $1203 \pm 24$ & $27.4 \pm 3.8$ & $25640 \pm 600$ & $937 \pm 133$ \\
\hline & $\mathrm{C}$ IV (doublet) & 1549.0 & $6799.58 \pm 0.72$ & $3.3897 \pm 0.0005$ & $10.4 \pm 1.7$ & $460 \pm 75$ & $21.6 \pm 2.9$ & $2590 \pm 420$ & $120 \pm 25$ \\
\hline & $\mathrm{He}$ II & 1640.5 & $\ldots$ & & $\ldots$ & $\ldots$ & & $\ldots$ & \\
\hline \multirow[t]{5}{*}{ 4C 60.07 (core) } & Ly $\alpha$ & 1215.7 & $5826.52 \pm 0.07$ & $3.7928 \pm 0.0001$ & $47.1 \pm 0.2$ & $2424 \pm 12$ & $\leq 0.01$ & $41.1 \pm 0.2$ & $\geq 5000$ \\
\hline & $\mathrm{C}$ IV & 1548.2 & $7415.7 \pm 0.37$ & $3.7899 \pm 0.0004$ & 81.4 & $6 \times 900$ & $\ldots$ & $\ldots$ & $\ldots$ \\
\hline & $\mathrm{C}$ IV & 1550.8 & $7428.1 \pm 0.37$ & $3.7899 \pm 0.0004$ & $\ldots$ & $\ldots$ & $\ldots$ & $\ldots$ & $\ldots$ \\
\hline & He II & 1640.5 & $7855.7 \pm 1.07$ & $3.7887 \pm 0.0007$ & $73.9 \pm 2.7$ & $2820 \pm 105$ & $\leq 0.01$ & $9.5 \pm 0.3$ & $\geq 790$ \\
\hline & $\mathrm{C}$ III] & 1908.7 & $9140.7 \pm 1.70$ & $3.7889 \pm 0.0007$ & & & & $\ldots$ & \\
\hline \multirow[t]{4}{*}{ 4C 60.07 (total) } & Ly $\alpha$ & 1215.7 & $5824.59 \pm 0.18$ & $3.7913 \pm 0.0002$ & $44.9 \pm 0.5$ & $2310 \pm 25$ & $\leq 0.01$ & $56.1 \pm 0.7$ & $\geq 4000$ \\
\hline & C IV (doublet) & 1549.0 & $7420.75 \pm 1.34$ & $3.7907 \pm 0.0009$ & $36.5 \pm 3.3$ & $1475 \pm 130$ & $\leq 0.01$ & $5.0 \pm 0.3$ & $\geq 600$ \\
\hline & $\mathrm{He}$ II & 1640.5 & $7848.50 \pm 4.41$ & $3.7843 \pm 0.0027$ & 84 & $3220 \pm 4400$ & $\leq 0.02$ & $4.0 \pm 1.6$ & $\geq 230$ \\
\hline & $\mathrm{C}$ III] & 1908.7 & $9141.88 \pm 1.82$ & $3.7896 \pm 0.0010$ & $59.9 \pm 4.7$ & $1960 \pm 155$ & $\leq 0.01$ & $0.2 \pm 0.8$ & $\geq 14$ \\
\hline 4C 60.07 (filament) & Ly $\alpha$ & 1215.7 & $5818.07 \pm 0.29$ & $3.7859 \pm 0.0002$ & $20.0 \pm 0.7$ & $1030 \pm 36$ & $\leq 0.01$ & $6.2 \pm 0.2$ & $\geq 6600$ \\
\hline 4C 60.07 (nearby galaxy) .................... & {$[\mathrm{O}$ II $]$} & 3727.0 & $6973.30 \pm 0.33$ & $0.8710 \pm 0.0001$ & $30.0 \pm 0.8$ & $1290 \pm 34$ & $\leq 0.06$ & $9.0 \pm 0.1$ & $\geq 140$ \\
\hline 4C 60.07 (galaxy at filament)........... & {$[\mathrm{O}$ II $]$} & 3727.0 & $7046.98 \pm 4.25$ & $0.8908 \pm 0.0011$ & $51.7 \pm ?$ & $2200 \pm 450$ & $\leq 0.02$ & $3.7 \pm 0.2$ & $\geq 180$ \\
\hline \multirow[t]{9}{*}{ 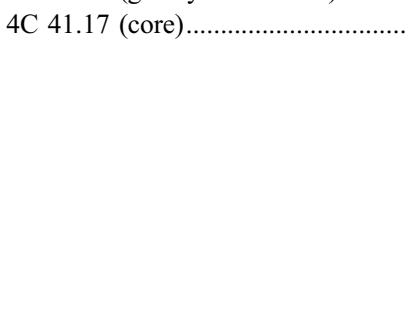 } & $\operatorname{Ly} \alpha$ & 1215.7 & $5834.5 \pm 0.1$ & $3.8002 \pm 0.0001$ & $\ldots$ & $613 / 1373 \pm 13 / 45$ & $\ldots$ & $146 \pm 5.5$ & $\ldots$ \\
\hline & $\mathrm{C}_{\text {IV }}$ & 1548.2 & $7428.9 \pm 0.2$ & $3.7984 \pm 0.0001$ & $\ldots$ & $541 \pm 14$ & $\ldots$ & $7.5 \pm 0.2$ & $\ldots$ \\
\hline & $\mathrm{C}$ IV & 1550.8 & $7441.2 \pm 0.2$ & $3.7984 \pm 0.0001$ & $\ldots$ & $541 \pm 14$ & $\ldots$ & $5.7 \pm 0.2$ & $\ldots$ \\
\hline & $\mathrm{He}$ II & 1640.5 & $7870.7 \pm 0.4$ & $3.7979 \pm 0.0002$ & $\ldots$ & $553 \pm 28$ & .. & $5.5 \pm 0.3$ & $\ldots$ \\
\hline & $\mathrm{C}$ III] & 1908.7 & $9152.5 \pm 0.6$ & $3.7951 \pm 0.0003$ & $\ldots$ & $511 / 1120 \pm 151 / 135$ & & $9.1 \pm 0.2$ & $\ldots$ \\
\hline & {$[\mathrm{O}$ II $]$} & 3727.0 & $17890.36 \pm 0.54$ & $3.8002 \pm 0.0001$ & $42.4 \pm 1.1$ & $711 \pm 18$ & $57.8 \pm 7.1$ & $\ldots$ & $\ldots$ \\
\hline & $\mathrm{H} \beta$ & 4861.0 & $23351.72 \pm 3.27$ & $3.8039 \pm 0.0007$ & $33.0 \pm 8.1$ & $424 \pm 104$ & $0.19 \pm 0.04$ & $17.2 \pm 4.9$ & $92.7 \pm 32.9$ \\
\hline & [O III] & 4959 & $23816.29 \pm 1.20$ & $3.8026 \pm 0.0002$ & $36.1 \pm 3.0$ & $454 \pm 38$ & $0.18 \pm 0.04$ & $59.8 \pm 5.2$ & $337.8 \pm 81.2$ \\
\hline & {$[\mathrm{O}$ III] } & 5006.9 & $24046.74 \pm 0.42$ & $3.8027 \pm 0.0001$ & $37.9 \pm 1.1$ & $472 \pm 13$ & $0.18 \pm 0.04$ & $222.8 \pm 5.4$ & $1260 \pm 295$ \\
\hline \multirow[t]{3}{*}{ 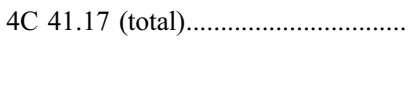 } & $\mathrm{H} \beta$ & 4861 & $23355.84 \pm 8.35$ & $3.8047 \pm 0.0017$ & 55 & $700 \pm 270$ & $0.47 \pm 0.12$ & $52.5 \pm 21.5$ & $112 \pm 54$ \\
\hline & {$[\mathrm{O}$ III] } & 4959 & $23810.62 \pm 3.03$ & $3.8015 \pm 0.0006$ & $50.8 \pm 7.8$ & $640 \pm 98$ & $0.50 \pm 0.14$ & $129.4 \pm 20.5$ & $259 \pm 85$ \\
\hline & {$[\mathrm{O}$ III $]$} & 5006.9 & $24041.56 \pm 1.13$ & $3.8017 \pm 0.0002$ & $58.4 \pm 3.0$ & $728 \pm 37$ & $0.47 \pm 0.16$ & $484.7 \pm 22.6$ & $1031 \pm 351$ \\
\hline
\end{tabular}

Nоте.-Where two values are given, the line is split. 
self-absorption feature on the blue side of the profile centered at $\lambda=5331.58(z=3.3857)$, which can be fitted with a Voigt profile with a column density of $1.8 \times 10^{14} \mathrm{~cm}^{-2}$ and a Doppler parameter of $b=195 \pm 11 \mathrm{~km} \mathrm{~s}^{-1}$. The inferred blueshift is $\approx 100 \mathrm{~km} \mathrm{~s}^{-1}$. This cannot be the same gas that causes $\mathrm{H} \mathrm{I} \mathrm{ab-}$ sorption against the radio continuum, since Briggs et al. (1993) and Cody $\&$ Braun (2003) infer a redshift $z_{\mathrm{abs}}=3.3962$ with $N_{\mathrm{H}}=3 \times 10^{21} \mathrm{~cm}^{-2}$ and FWHM $=120 \mathrm{~km} \mathrm{~s}^{-1}$ for the absorbing gas. It is probable that the dense gas giving the $\mathrm{H}$ I absorption lies close to the nucleus, and any absorption that this may have produced in the Ly $\alpha$ line is completely veiled by the frequency redistribution produced by multiple scattering events.

The Ly $\alpha$ absorption can possibly be identified with a galaxywide outflow of material. In this respect, it is very similar to the galaxy-wide outflow identified by Wilman et al. (2005) in a Ly $\alpha$ galaxy associated with the SSA-22 protocluster. We return to this point in $\S 4$.

The underlying Ly $\alpha$ emission profile revealed by Figure 4 is very interesting, as it shows evidence of a strong velocity shear amounting to nearly $1000 \mathrm{~km} \mathrm{~s}^{-1}$ across the central arcsecond. The upper (northeast) component has the typical triangular shape resulting from spatially extended absorption that is blueshifted relative to the systemic velocity (see Dey 1999), and it is terminated by the self-absorption on the blue side. The lower (southwest) component may well have an underlying shape which is the symmetrically reversed version of the northeast component, but the self-absorption makes this difficult to trace, and the line reaches its maximum brightness at a relative velocity of $-500 \mathrm{~km} \mathrm{~s}^{-1}$ as it emerges from under the absorption. Because of the self-absorption, a Gaussian fit to the line profile is not valid and yields spuriously large values, as can be seen in Figure 7.

The C IV $\lambda 1549$ emission is both spatially extended and broad $\left(\approx 1000 \mathrm{~km} \mathrm{~s}^{-1}\right)$ and is centered on the nucleus. The line broadening in the nucleus is probably, once again, the result of a direct interaction between the relativistic outflow associated with the peculiar radio source (see Carilli 1995 for details) and its dense cocoon of ISM.

\section{DISCUSSION}

\subsection{Emission-Line Diagnostics}

The relative intensities of emission lines are, in principle, powerful diagnostic tools for studying the excitation mechanisms, metallicities, and physical conditions in the emission-line gas associated with AGNs. Diagnostic diagrams for optical-line ratios have been most extensively used to probe the gas in nearby active galaxies, where evidence for both jet- and accretionpowered shocks and for photoionization by the central AGN has been adduced (e.g., Bicknell et al. 1997; Dopita et al. 1997; Groves et al. 2004a, 2004b). Consequently, these relationships have been best calibrated for rest-frame optical lines. However, line diagnostic diagrams applicable to both shock-ionized and photoionized AGNs have been developed for use in the UV (Allen et al. 1998; Groves et al. 2004b).

Until recently, for most HzRGs (De Breuck et al. 2000) or for the $3 \mathrm{C}$ radio galaxies (Best et al. 2000a, 2000b; Inskip et al. 2002a, 2002b) only the rest-frame UV lines have been available. The optical-line diagnostics have previously not been commonly used because of calibration difficulties and the limited sensitivity of near-IR spectrographs on telescopes of the 2-4 m class.

We would like to be able to use both UV and optical emissionline diagnostics to determine the mechanism that is responsible for ionizing these extended emission-line nebulae. The most likely candidates are photoionization by radiation from an AGN or stars, shock heating, and shocks with precursors that photoionize the region ahead of the shock by radiation from the gas. Studies of $z \approx 1$ radio galaxies have shown that the dominant ionization mechanism may depend on the evolutionary state of the radio source (e.g., Best et al. 2000a, 2000b; Inskip et al. 2002a, 2002b). Generally, it was found that the emission-line gas of small (i.e., young) sources is shock-ionized, but as the radio source expands beyond the host galaxy, interactions with the gas decrease and photoionization by the AGN takes over. As De Breuck et al. (2000) noted, for HzRGs the situation may be more complex.

Before we investigate these line diagnostics, we first use global energy requirements to constrain important source parameters on the basis of both photoionization and shock models. The total Ly $\alpha$ fluxes of the extended emission-line regions are of order $10^{-14} \mathrm{ergs}^{-1} \mathrm{~cm}^{-2}$, yielding luminosities $L_{\mathrm{Ly} \alpha} \simeq 10^{45} \mathrm{ergs} \mathrm{s}^{-1}$ (Reuland et al. 2003). If the source of ionization is an active nucleus, then the luminosity of the source derives either from the UV photons it produces or from the mechanical energy of the jets it powers. These luminosities can be derived from the theoretical ratio of the Ly $\alpha$ flux to the total flux in both shock and photoionization models. To do this we use the shock models from Dopita \& Sutherland (1996) and assume that the $P d V$ work implied by this luminosity is the mechanical energy flux of the jet. For the justification of this assumption, see Bicknell et al. (1997). For photoionization, we use the radiation-pressure-dominated photoionization models from Groves et al. (2004a, 2004b), assuming a power-law spectrum with index $\alpha=-1.4\left(f_{\nu} \approx \nu^{\alpha}\right)$. From these we derive either an ionizing radiative or a mechanical energy flux of at least $1.1 \times 10^{46} \mathrm{ergs} \mathrm{s}^{-1}$. This flux is consistent with a typical luminous embedded QSO.

Alternatively, some of the ionization may be due to young stars such as are found in the numerous clumpy components of the southern region of 4C 41.17 (van Breugel et al. 1999) and whose formation has probably been triggered in the dense cocoon surrounding the central radio jet (Dey et al. 1997; Bicknell et al. 2000). In this case, assuming a stellar radiation field with an effective temperature of $42,000 \mathrm{~K}$, we infer (using the Kennicutt 1998 calibration) that a star formation rate of at least $3000 M_{\odot} \mathrm{yr}^{-1}$ would be needed to ionize the halo. This star formation rate is remarkably close to what is inferred from rest-frame far-IR observations (Dunlop et al. 1994; Stevens et al. 2003). However, this does not necessarily imply that stellar photoionization is the dominant ionization mechanism. Photoionization by stars usually results in Ly $\alpha$ equivalent widths less than $240 \AA$ (assuming stellar populations at solar metallicity; Charlot \& Fall 1993) and would seem inconsistent with the large equivalent widths observed ( $\approx 500 \AA$ A Dey et al. 1997). The strength of the C IV lines and other high-excitation species also militates against a purely stellar origin of the ionizing flux. Indeed, Bicknell et al. (2000) have used the emission spectrum to infer that shocks are the dominant source of ionization in this region, and they have used the flux to determine the $P d V$ work being done by the relativistic jet. However, away from the radio axis photoionization by stars may well become more important. The galaxy $4 \mathrm{C} 41.17$ is a young system, and recent modeling suggests that any primordial stellar populations could be very efficient in emitting significant amounts of ionizing flux while remaining virtually undetectable in the optical (see Fosbury et al. 2003; Panagia et al. 2003).

Using the observed line ratios, better constraints on the ionizing process can be derived. Dey et al. (1997) determined the following ratios for the narrow rest-frame UV lines of $4 \mathrm{C} 41.17$ : 


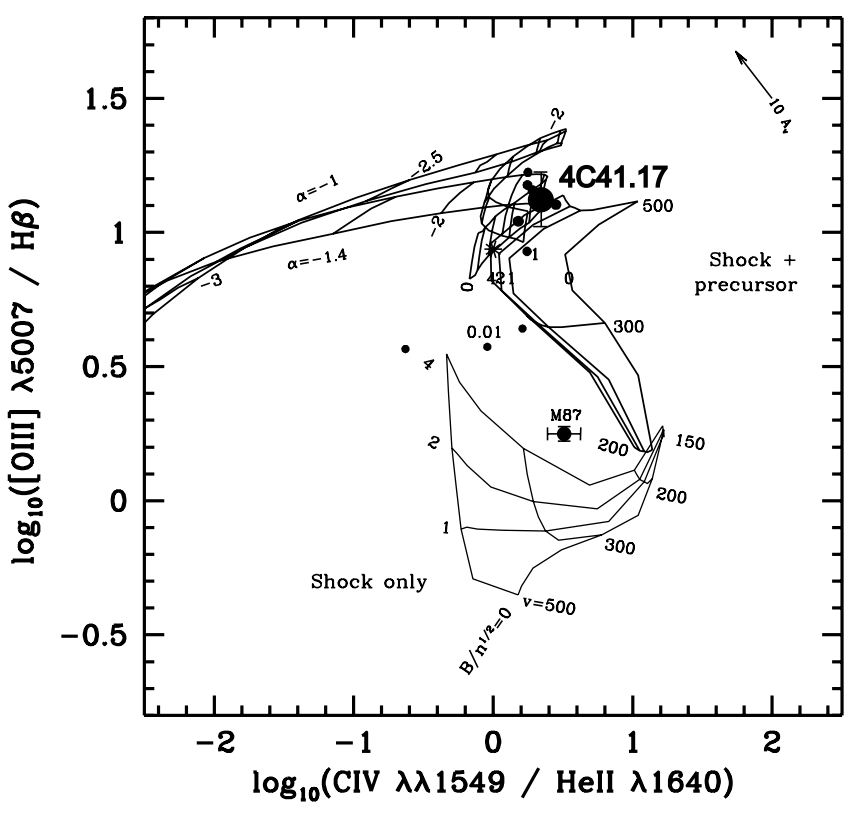

FIG. 11. - UV-optical line diagnostic diagram adapted from Allen et al. (1998). The ratios $[\mathrm{O} \mathrm{III}] / \mathrm{H} \beta$ and $\mathrm{C}$ IV/ $\mathrm{He}$ II are used to separate shock (lower part of panel), shock+ precursor (right-hand side), and photoionization (upper left) models for solar metallicity gas. The shock models are marked according to the shock velocity and magnetic parameter, and the photoionization models according to the ionization parameter, $U$, and the power-law index of the photoionizing radiation field $(\alpha)$. The circles represent nearby $z \approx 1$ galaxies, and the accretion disk of M87 is explicitly identified. The large circle with error bars represents the nucleus of $4 \mathrm{C} 41.17$. This proves that the excitation of $4 \mathrm{C} 41.17$ is similar to that of other radio galaxies and, like them, is shock-dominated.

$\mathrm{C}_{\text {III }} / \mathrm{C}_{\text {IV }} \approx 0.14(\approx 0.7$ for total fluxes $)$ and $\mathrm{C}$ IV $/ \mathrm{He}_{\text {II }} \approx 2.4$. The latter is close to the maximum of 3.1 predicted by photoionization models. Although the narrow emission-line strengths of C III], C IV, and He II can be matched by a simple nuclear AGN photoionization model with solar metallicity clouds, a highionization parameter $U \lesssim 0.1$, and a power-law ionizing source with index $\alpha=-1.5$, Dey et al. (1997) and Bicknell et al. (2000) argue that the dominant ionizing mechanism is shocks rather than photoionization by the nucleus.

Figure 11 shows that a combination of rest-frame UV ( $\mathrm{C}$ IV $\lambda 1549 / \mathrm{He}$ II $\lambda 1640)$ and rest-frame optical ([O III] $25007 / \mathrm{H} \beta$ ) emission-line flux ratios can help to separate pure photoionization from shock-dominated mechanisms. This diagram was derived assuming solar metallicity gas and is relatively insensitive to the effects of dust extinction as the ratios are determined from lines close in wavelength. Iwamuro et al. (2003) conducted a rest-frame UV-optical emission-line study of 15 radio galaxies with $2<z<$ 2.6. They found that there is a range in the observed line ratios suggesting that some objects are best explained by photoionization of low-metallicity gas, while others are consistent with the shock+ precursor model. Carson et al. (2001) and Maxfield et al. (2002) found evidence for changing line ratios within sources, suggesting that the dominant ionization process depends on the region of interest.

Based on previously published values of $[\mathrm{O}$ III $] / \mathrm{H} \beta \approx 3.4$ and 2.8 for 4 C 41.17 and B2 $0902+34$, respectively (Eales \& Rawlings 1993), it seemed that pure photoionization models could be ruled out. However, the $\mathrm{H} \beta$ detections were marginal, and using the total integrated line fluxes for $4 \mathrm{C} 41.17$ from the present study we derive ratios of $[\mathrm{O}$ III $] \lambda 5007 / \mathrm{H} \beta \approx 9.6$ for the central region and $[\mathrm{O} \mathrm{III}] \lambda 5007 / \mathrm{H} \beta \approx 11.8$ for the spatially integrated spectrum that are much higher and are consistent with both pure photoionization and shock + precursor models. The point

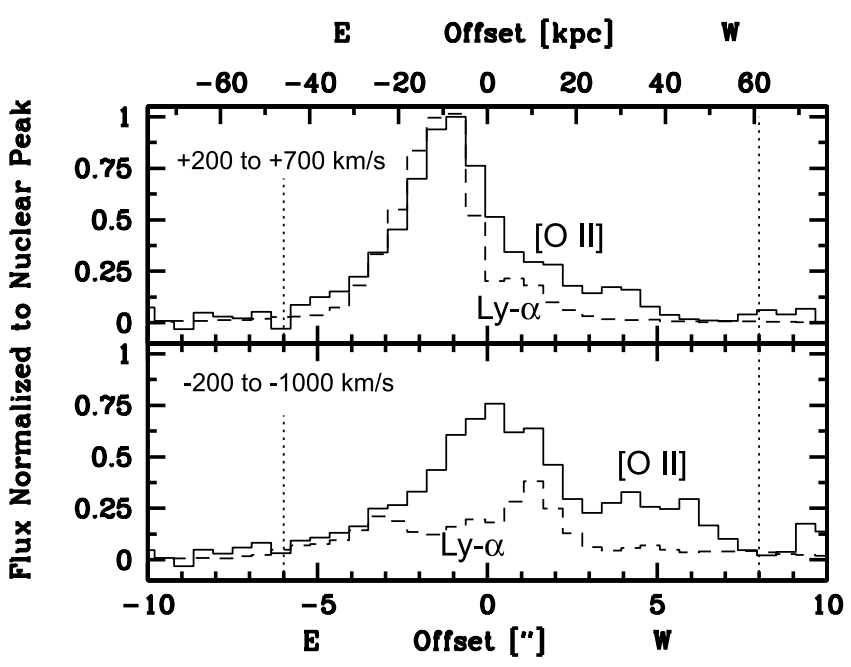

FIG. 12.-Top: Normalized surface brightness profiles of the [O II] (solid line) and Ly $\alpha$ (dashed line) emission along the inner radio axis and southwest filament of $4 \mathrm{C} 41.17$. These are integrated in the velocity range +200 to $+700 \mathrm{~km} \mathrm{~s}^{-1}$ and clearly show the spatially extended [O II] emission. The spatial zero point corresponds to the position of the radio core. Bottom: Same as top, but for the velocity range -200 to $-1000 \mathrm{~km} \mathrm{~s}^{-1}$. The [O II] emission is detected out to $\approx 8^{\prime \prime}(\approx 60 \mathrm{kpc})$ west of the nucleus, where it is blueshifted by $\approx-600 \mathrm{~km} \mathrm{~s}^{-1}$ relative to the He II line. The projected distances of the southwest and northeast radio lobes along the slit direction are indicated (dotted lines). The +200 to $-200 \mathrm{~km} \mathrm{~s}^{-1}$ range for [O II] is confused by near-IR skylines and is therefore not shown.

derived for the spatially integrated spectrum of $4 \mathrm{C} 41.17$ is also shown in Figure 11. This appears to show that the excitation of $4 \mathrm{C} 41.17$ is similar to that of other radio galaxies. It cannot be due predominantly to stars, for which the $[\mathrm{O}$ III $] / \mathrm{H} \beta$ would be of order 3 and both C IV $\lambda 1549$ and He II would be very faint.

The extended regions of $4 \mathrm{C} 41.17$ have a much lower excitation, however. As discussed in $\S 3.1 .1$, for $4 \mathrm{C} 41.17$, [O II] emission was detected as far as $\approx 60 \mathrm{kpc}$ from the nucleus. Figure 12 shows the velocity-integrated relative intensities of Ly $\alpha$ and [O II] as a function of distance from the nucleus for a redshifted $(+200$ to $\left.+700 \mathrm{~km} \mathrm{~s}^{-1}\right)$ and a blueshifted $\left(-200\right.$ to $-1000 \mathrm{~km} \mathrm{~s}^{-1}$ ) velocity interval. The redshifted [O II] flux closely follows that of the Ly $\alpha$, while the blueshifted [O II] shows a relative enhancement over the range $3^{\prime \prime}-8^{\prime \prime}$ west of the nucleus. In this region no evidence for [O III] is found (Fig. 2), whereas the [O III] emission in the nuclear region is much brighter than the [O II]. This shows that the $\left[\mathrm{O}_{\text {III }}\right] /\left[\mathrm{O}_{\text {III }}\right]$ ratio is much lower $(\approx 1-2)$ in the outer regions than in the center $(\approx 3-4)$, implying that a different mechanism is responsible for the extended [O II] emission. The $[\mathrm{O}$ III]/[O II] ratio is known to be sensitive to the ionization parameter in the nebula. Because they have higher ionization parameters and central stars with higher effective temperatures for a given age, the lowmetallicity $\mathrm{H}$ II regions are characterized by higher $[\mathrm{O}$ III $] /[\mathrm{O}$ II] ratios. Since this ratio falls to very low values in the outer parts of $4 \mathrm{C} 41.17$ this proves that Population III stars with their very high effective temperatures cannot be responsible for the excitation of the outer regions. Excitation by shocks is a more likely ionizing source, since the emission is extended along the radio axis and both the $\mathrm{Ly} \alpha$ and $[\mathrm{O}$ II $]$ are blueshifted by $\approx 600 \mathrm{~km} \mathrm{~s}^{-1}$. Furthermore, the peak in the [O II] emission on the west side of 4C 41.17 corresponds to the point at which the velocity dispersion drops steeply near the boundary of the outer radio lobe. This suggests that the [O ${ }_{\text {II }}$ is excited by shocks in an outward-propagating cocoon around the radio lobe. We note here that $[\mathrm{O}$ II] emission is particularly strong in shocks. This corroborates the suggestions by Dey et al. (1997) and Bicknell et al. (2000) that the Ly $\alpha$ 
emission, at least in this direction, is not due to scattering but that the nebula must be locally ionized by shocks related to the radio source. Scharf et al. (2003) proposed that the escape of X-ray emission from the shocked region could be responsible for ionizing the extended halo beyond the radio lobes.

From the jet shock spectrum, Bicknell et al. (2000) inferred that the metallicity of the star-forming cocoon around the radio jet was about the same as for the LMC: one-half to one-third of solar. For the nucleus itself, $\mathrm{N} v$ is often used as a tracer of the chemical abundance, because it is rather insensitive to shock ionization, density fluctuations, and ionization parameter, and as a secondary nucleosynthesis element has a quadratic dependence on metallicity (Villar-Martín et al. 1999; Groves et al. 2004a). This would imply that that would only be detected in highly chemically enriched sources and not significant in the majority of HzRGs (Röttgering et al. 1997; De Breuck et al. 2000). Vernet et al. (2001) presented a metallicity sequence for HzRGs that is similar to the one for quasars (Hamann \& Ferland 1993). They included 4C 41.17 in their study, and a metallicity of $Z \approx 1.3 Z_{\odot}$ was inferred for the central region (aperture of $2^{\prime \prime} \times 1^{\prime \prime}$; Dey et al. 1997).

Thus, the nuclear and jet cocoon spectra of 4C 41.17 argue for a fair degree of chemical enrichment, perhaps more in the nucleus than in the jet region. However, the halo region is by no means primordial in its abundances. Although we cannot directly infer the actual metallicity, we can say that the detection of strong [O II] emission out to $\approx 60 \mathrm{kpc}$ from the nucleus demonstrates that the halo has been chemically enriched throughout its volume, at least in the illuminated regions.

A similar conclusion is also inferred for 4C 60.07. The very similar velocity morphologies and spatial extent of the Ly $\alpha$ and [O II] spectra at P.A. $=81^{\circ}$ seen in Figure 3 once again show that the enriched gas is distributed throughout the Ly $\alpha$ halo. The strongly $\mathrm{C}$ IV-emitting shell in the nuclear vicinity shows that this galaxy too is excited primarily by the AGN rather than by star formation. The expanding shell morphology of both Ly $\alpha$ and $\mathrm{C}$ IV further argues that $4 \mathrm{C} 60.07$, like 4C 41.17, is excited mostly by shocks in the dense expanding cocoon of interstellar gas pushed by the relativistic jets from the nucleus.

\subsection{Galaxy Mass Estimates}

The HzRGs are known to represent some of the most massive galaxies formed in the early universe. An estimate of their mass, by any means, is important to constrain the $\Lambda$ CDM models of galaxy formation, since the formation of such massive galaxies so early on might present something of an issue for the theory to resolve. We therefore provide these mass estimates in this section.

\subsubsection{Luminosity-based Masses}

Van Breugel et al. (1999) estimated the mass of 4C 41.17 using the integrated rest-frame UV light. They found it to be a very massive galaxy with $L_{\mathrm{UV}} \approx 17 L^{*}$. Similarly, Graham et al. (1994) used near-IR imaging with the line-free $K_{S}$ filter to estimate the mass of the galaxy directly. In a $4^{\prime \prime}$ diameter aperture they found $K_{S}=19.6 \pm 0.6 \mathrm{mag}$. This converts in their cosmology to an absolute magnitude for $4 \mathrm{C} 41.17$ of $M_{B}=-23.0$, or about $20 L^{*}$.

A lower mass limit for the ionized gas in the halo can be obtained from photoionization modeling of the total Ly $\alpha$ flux, neglecting the effect of dust absorption, and assuming that the ISM is fully ionized. From this we derive an average thermal electron density in the halo of $n_{e \text {, halo }}=0.1 f_{v}^{-1 / 2} \mathrm{~cm}^{-3}$, where $f_{v}$ is the volume filling factor of the $\mathrm{Ly} \alpha$-emitting gas. This yields a mass of gas of $M_{\text {halo }} \approx 6 \times 10^{12} f_{v}^{1 / 2} M_{\odot}$. For a volume filling factor $f_{v} \approx 1$ the inferred amount of gas is substantial and comparable to the broadband mass estimates of the host galaxy and of fully formed, massive elliptical galaxies in the local universe. However, the fact that the Ly $\alpha$ line appears to provide a good dynamical mass tracer and is not grossly affected by self-absorption argues in favor of $f_{v} \ll 1$ and, consequently, for a much lower total mass. The molecular component of $4 \mathrm{C} 41.17$ has recently been traced by De Breuck et al. (2005), who have detected the $\mathrm{CO}(4-3)$ transition using the IRAM interferometer. They find two massive $\mathrm{CO}$ clouds $\left(M_{\mathrm{dyn}}^{\text {rot }} \approx 6 \times 10^{10} M_{\odot}\right)$ which coincide with two different dark lanes located close to the nucleus in the deep Ly $\alpha$ image. This is similar to the CO content of the submillimeter galaxies, as measured by Greve et al. $\left(2005 ; M_{\mathrm{CO}} \approx\right.$ $3 \times 10^{10} M_{\odot}$ ), suggesting once again that the HzRGs and the submillimeter galaxies are drawn from similar populations of galaxies, differing only in the activity of their AGNs.

\subsubsection{Dynamical Mass Estimates}

Van Ojik et al. (1997) and Villar-Martín et al. (2003) have presented mass estimates for the more quiescent parts of the emission-line regions in their studies. There are two methods of estimating the dynamical masses. First, one can assume that the halos consist of gas that has settled in rotating disks. Second, the halos can be envisaged as consisting of virialized clumps, which have velocity dispersions that balance the gravitational forces.

In the case of rotating disks, the mass can be estimated by measuring the velocity shear across the halo and using $M_{\mathrm{dyn}}^{\mathrm{rot}}=$ $R V^{2} / G \sin ^{2} i$, where $R$ is the radius of the disk, $V$ is half the amplitude of the rotation curve, and $i$ is the inclination of the disk with respect to the plane of the sky. Villar-Martín et al. (2003) found evidence for rotation in $\approx 50 \%$ of the objects they looked at and inferred masses of order $M_{\mathrm{dyn}}^{\mathrm{rot}}\left(\sin ^{2} i\right) \approx(0.3-3) \times 10^{12} M_{\odot}$.

Looking at our measured relative velocity distributions for 4C 41.17 , we infer a velocity shear of $\approx 300 \mathrm{~km} \mathrm{~s}^{-1}(+100$ to $-200 \mathrm{~km} \mathrm{~s}^{-1}$ ) at P.A. $=76^{\circ}$, over a distance of $R=$ $20^{\prime \prime} / 2=77 \mathrm{kpc}$. From this we infer a dynamical mass of about $M_{\text {dyn }}^{\text {rot }}\left(\sin ^{2} i\right) \approx 4 \times 10^{11} M_{\odot}$. The velocity profiles at P.A. $=19^{\circ}$ and $21^{\circ}$ show no signs of a shear, consistent with their approximate alignment along the axis of rotation.

The observation for 4C 60.07 does not show an indication of being settled in a disk. Rather, it could be supported against gravitation by velocity dispersions of the cloudlets in the halo. For this scenario the dynamical mass is given by $M_{\mathrm{dyn}}^{\mathrm{vir}}=5 R V_{R}^{2} / G$, where $V_{R}$ is the radial velocity dispersion of the clouds. Using this relation we derive dynamical masses for the outer regions of $M_{\mathrm{dyn}}^{\mathrm{vir}}=(5-10) \times 10^{11} M_{\odot}$. For the inner regions, where the signal-to-noise ratio is better, we find a lower mass of $M_{\mathrm{dyn}}^{\mathrm{vir}} \approx 2 \times 10^{11} M_{\odot}$.

The Ly $\alpha$ emission profile of B2 $0902+34$ revealed by Figure 4 suggests a strong nuclear velocity shear. If this is interpreted as a rotation, then it would imply a rotational velocity of $\approx 550 \mathrm{~km} \mathrm{~s}^{-1}$ over a distance of $3^{\prime \prime}$ or a rotation velocity of $\approx 275 \mathrm{~km} \mathrm{~s}^{-1}$ at $R \approx 12 \mathrm{kpc}$. This implies a dynamical mass within this core region of $M_{\mathrm{dyn}}^{\text {rot }}\left(\sin ^{2} i\right) \approx 2 \times 10^{11} M_{\odot}$. However, as discussed in $\S 3.1 .7$, this velocity gradient could equally well be due to the expansion of a shell of interstellar gas driven by a directed bipolar outflow from the AGN with a velocity $v_{\text {exp }}$.

\section{OUTFLOWS}

Absorbing gas associated with lower velocity outflows has now been observed in several HzRGs, almost all of which show asymmetric Ly $\alpha$ profiles suggesting the presence of blueshifted absorbing gas which appears to be spatially extended over the 
entire emission-line region (see, e.g., van Ojik et al. 1997; Dey 1999; Jarvis et al. 2003; Wilman et al. 2004). Spectroscopic evidence for outflows at high redshifts also exists for other, presumably less massive, galaxies (Pettini et al. 2001; Dawson et al. 2002).

There is an ongoing debate whether massive $\left(>L_{*}\right)$ galaxies or less massive $\left(<L_{*}\right)$ galaxies enrich the intracluster medium. Martin (1999) and Heckman et al. (2000) found that outflow speeds, $v_{\text {exp }}$, are largely independent of galaxy mass. This would imply that smaller galaxies are more efficient at ejecting enriched material to large radii. Specifically, Heckman (2002) claimed that for massive galaxies the metals will not escape the deep potential wells. However, recent modeling suggests that the bulk of the metals in clusters are produced by $L_{*}$ and brighter galaxies (e.g., Nagashima et al. 2005).

For all three HzRGs we have observed, we found features that can be interpreted as symptomatic of outflows. The extended optical filament of 4C 60.07 is consistent with an outflow in the plane of the sky. This galaxy also shows crescent-shaped arcs around the radio core and an unobscured optical nucleus that are consistent with an expanding cocoon; see Figure 8. The blueshifted carbon-rich component of B2 $0902+34$ seems to point toward an outflow of enriched material with an outflow velocity, $v_{\text {exp }}$, of up to $1000 \mathrm{~km} \mathrm{~s}^{-1}$. The near-IR [O II] spectroscopy (Figs. 2, 5 , and 12) and the earlier optical Ly $\alpha$ spectroscopy of 4C 41.17 (Fig. 2; Dey et al. 1997) show that both emission lines exhibit large blueshifted radial velocities of $\approx 600-900 \mathrm{~km} \mathrm{~s}^{-1}$ seen in projection along the radio axis. In particular, the gas is very disturbed along the southwest filament, with $\operatorname{Ly} \alpha$ velocity widths ranging up to $\Delta v_{F} \approx 900-1600 \mathrm{~km} \mathrm{~s}^{-1}$. Beyond the radio hot spot, the velocity and velocity widths decrease abruptly. The kinematics, its radial filamentary structure, and chemical enrichment of the gas all indicate a process of entrainment of material away from the central regions by the radio jet. In this scenario, the optical filament represents the shocked radiative cocoon of the radio source.

For a galaxy mass of $M_{\text {gal }} \sim 1 \times 10^{12} M_{\odot}$ within a radius of $R \sim 40 \mathrm{kpc}$, figures that seem to be typical for these HzRGs, the escape velocity $v_{\mathrm{esc}}=\left(2 G M / R_{\mathrm{gal}}\right)^{1 / 2}$ is of order $\sim 450 \mathrm{~km} \mathrm{~s}^{-1}$. The velocity gradients found from the spectroscopic observations are a factor of 1.5-2.5 larger than this and imply that the ejection of the ISM originating from close to the nucleus of such galaxies is a viable scheme to both chemically enrich the intergalactic medium and to terminate the epoch of star formation in these massive galaxies.

While it may still be true that purely starburst-powered supergalactic winds may not be sufficiently energetic for enriched nuclear material to escape from the galaxian potentials, the additional driving force produced by the energy content of the relativistic lobes produced by the central AGN seems quite able to blow matter out of the potential of the galaxy. This is direct evidence of the operation of the type of feedback process proposed by Silk \& Rees (1998). In this process, the growth of the supermassive central black hole is terminated by the ejection of the reservoir of interstellar gas, once the mass of the black hole exceeds a critical value. In their model, this critical value is determined by the relationship between the radiation pressure and the depth of the stellar potential, and it is able to provide a qualitative description of the Magorrian relationship (Magorrian et al. 1998), or the relationship between the mass of the central black hole and the velocity dispersion of the bulge (Magorrian et al. 1998; Ferrarese \& Merritt 2000; Gebhardt et al. 2000).

The observations presented here suggest that this model needs to be modified somewhat. Rather than being driven by radiation pressure, the dynamical evidence is that they are driven by the
$P d V$ work done on the galactic medium by the relativistic jets, and that the ejection is occurring in the faster moving material in the shocked cocoon around the radio jet. Such a scheme is also suggested by the hydrodynamical simulations of Springel et al. (2005). However, rather than being ejected isotropically in a disklike galaxy, as in their model, in the HzRGs directional jets are being driven into a more spheroidal distribution of galactic interstellar gas. Given that 4C 41.17 has clear evidence of large amounts of shock-triggered star formation in this shocked cocoon, we can conclude that in the HzRGs we are witnessing the fireworks that terminate the epoch of star formation in these massive galaxies - the last hurrah before these galaxies become the massive "red and dead" elliptical galaxies we find today in the cores of the most massive clusters.

The multiple-component, asymmetric, and twisted radio structure of $4 \mathrm{C} 41.17$ suggests that during this phase the central SMBH has experienced multiple periods of radio source activity and precession (van Breugel et al. 1999; Steinbring et al. 2002). The precessing radio source will gradually evacuate the central region of the galaxy of its ISM. The timescales for star formation and radio source activity in 4C 41.17 are very similar (Chambers et al. 1990; Bicknell et al. 2000) and comparable to that for transporting the $[\mathrm{O}$ II] gas along the filament out to the vicinity of the southwest hot spot $\left(\approx 7 \times 10^{7} \mathrm{yr}\right.$ at $\left.\approx 900 \mathrm{~km} \mathrm{~s}^{-1}\right)$. It suggests an overall picture in which the initial growth of the galaxy through merging, the feeding and growth of the central black hole, the growth of the stellar component, and finally the triggering of SMBH the outflow and its associated starburst activity are all closely coupled processes.

\section{CONCLUSIONS}

Extended Ly $\alpha$ halos are observed both in HzRGs and in the submillimeter galaxies. Indeed, these objects may be drawn from the same underlying population, with the distinction that the HzRGs represent the radio-loud and active phase of the galaxian evolution. This seems to be supported by the observation that the largest radio-quiet Ly $\alpha$ halos have similar sizes and only slightly lower luminosities than the HzRGs but lack their large multicomponent continuum structures.

Our long-slit optical spectra of 4C 41.17, 4C 60.07, and B2 $0902+34$ have shown that their Ly $\alpha$ halos exhibit disturbed kinematics, with broad lines, large velocity shears, and, in some cases, expanding shells associated with the radio lobes. This clearly demonstrates that the relativistic jets are driving strong shocks into the galactic medium.

In $4 \mathrm{C} 41.17$ the near-IR spectra reveal very extended [O II] and $\left[\mathrm{O}_{\mathrm{III}}\right]$ emission distributed along the radio source axis, as far as $60 \mathrm{kpc}$ from the nucleus. This provides direct proof that the Ly $\alpha$ halos are both chemically enriched by star formation and ionized throughout the majority of their volume. The hypothesis that the Ly $\alpha$ is due to scattering off $\mathrm{H}$ I clouds is disproved. Likewise, the idea that the halo gas is primarily composed of chemically pristine "primordial" gas is also disproved. However, we cannot be certain that the gas in directions perpendicular to the radio axis contains a large primordial component, because the sensitivity of our spectroscopic observations is insufficient to detect oxygen lines at the low surface brightness expected. To settle this point would require very deep near-IR spectroscopy of the halo gas outside the radio sources, well beyond the hot spots and orthogonal to the radio axes. This would be a very difficult observation to make.

Our observations of HzRGs also help cast observational light on the origin of the correlation found in galaxies between the stellar velocity dispersion and the black hole mass (Gebhardt et al. 
2000; Ferrarese \& Merritt 2000). We find clear evidence of outflows of chemically enriched gas associated with the jets, and velocity dispersions in the expanding cocoons around the jets which probably exceed the escape velocity. In addition, B2 0902+34 shows that Ly $\alpha$ absorption can be identified with a galaxy-wide outflow of material, similar to the galaxy-wide outflow identified by Wilman et al. (2005). This demonstrates that in the HzRGs the central black hole has grown sufficiently to be able to profoundly modify its surrounding galactic medium, pushing it into outflow and (in the case of $4 \mathrm{C} 41.17$ ) triggering large quantities of star formation in the shocked galactic medium cocooning the radio lobes. Furthermore, the precession of the radio lobes seen in $4 \mathrm{C}$ 41.17 will ensure that eventually all of the ISM will be shocked and either be converted into stars or be ejected from the galaxy.

These observations can be understood in terms of the general picture of Silk \& Rees (1998) that feedback of the black hole on its host galaxy eventually limits the growth of the black hole. However, unlike the Silk \& Rees (1998) concept, the feedback process is not primarily radiation pressure but is the mechanical energy input delivered by the relativistic jets. Thus, in the HzRGs we may be observing the moment at which galaxy collapse gives way to mass ejection and a newly born galaxy is revealed. We may speculate that this is the defining moment at which such galaxies enjoy one last violent burst of shock-induced star formation before beginning their evolution to become the "red and dead" massive elliptical galaxies we see in our local universe.
We thank all the staff at the W. M. Keck Observatory for their excellent support. The authors wish to recognize and acknowledge the very significant cultural role and reverence that the summit of Mauna Kea has always had within the indigenous Hawaiian community. We are most grateful to have the opportunity to conduct observations from this mountain. M. R. thanks Mario Livio and the Space Telescope Science Institute for generous hospitality. This work was performed under the auspices of the US Department of Energy, National Nuclear Security Administration, by the University of California, Lawrence Livermore National Laboratory, under contract W-7405-Eng-48. W. v. B. acknowledges support for radio galaxy studies at the Institute for Geophysics and Planetary Physics at Lawrence Livermore National Laboratory and at UC Merced, including the work reported here, with the Hubble, Spitzer, and Chandra space telescopes via NASA grants HST GO-9779, GO-10127, SST GO-3482, SST GO3329, and Chandra/CXO GO-06701011. M. D. acknowledges the support of the ANU and the Australian Research Council (ARC) for his ARC Australian Federation Fellowship, as well as the ARC Discovery projects DP0208445 and DP0664434. This work was supported by the European Community Research and Training Network program, The Physics of the Intergalactic Medium. A. D.'s research is supported by NOAO, which is operated by the Association of Universities for Research in Astronomy, Inc., under a cooperative agreement with the National Science Foundation.

Aguirre, A., Hernquist, L., Schaye, J., Katz, N., Weinberg, D. H., \& Gardner, J. 2001, ApJ, 561, 521

Ahn, S. 2004, ApJ, 601, L25

Allen, M. G., Dopita, M. A., \& Tsvetanov, Z. I. 1998, ApJ, 493, 571

Athreya, R. M., Kapahi, V. K., McCarthy, P. J., \& van Breugel, W. 1998, A\&A, 329,809

Benson, A. J., Bower, R. G., Frenk, C. S., Lacey, C. G., Baugh, C. M., \& Cole, S. 2003, ApJ, 599, 38

Best, P. N., Röttgering, H. J. A., \& Longair, M. S. 2000a, MNRAS, 311, 1 2000b, MNRAS, 311, 23

Bicknell, G. V., Dopita, M. A., \& O'Dea, C. P. 1997, ApJ, 485, 112

Bicknell, G. V., Sutherland, R. S., van Breugel, W. J. M., Dopita, M. A., Dey, A., \& Miley, G. K. 2000, ApJ, 540, 678

Briggs, F. H., Sorar, E., \& Taramopoulos, A. 1993, ApJ, 415, L99

Carilli, C. L. 1995, A\&A, 298, 77

Carilli, C. L., Owen, F. N., \& Harris, D. E. 1994, AJ, 107, 480

Carilli, C. L., Röttgering, H. J. A., van Ojik, R., Miley, G. K., \& van Breugel, W. J. M. 1997, ApJS, 109, 1

Carson, J. E., et al. 2001, ApJ, 563, 63

Chambers, K. C., Miley, G. K., \& van Breugel, W. J. M. 1990, ApJ, 363, 21

Chambers, K. C., Miley, G. K., van Breugel, W. J. M., Bremer, M. A. R., Huang, J.-S., \& Trentham, N. A. 1996, ApJS, 106, 247

Chapman, S. C., Lewis, G. F., Scott, D., Richards, E., Borys, C., Steidel, C. C., Adelberger, K. L., \& Shapley, A. E. 2001, ApJ, 548, L17

Charlot, S., \& Fall, S. M. 1993, ApJ, 415, 580

Cody, A. M., \& Braun, R. 2003, A\&A, 400, 871

Dawson, S., Spinrad, H., Stern, D., Dey, A., van Breugel, W., de Vries, W., \& Reul, M. 2002, ApJ, 570, 92

De Breuck, C., Downes, D., Neri, R., van Breugel, W., Reuland, M., Omont, A., \& Ivison, R. 2005, A\&A, 430, L1

De Breuck, C., Röttgering, H., Miley, G., van Breugel, W., \& Best, P. 2000, A\&A, 362, 519

De Breuck, C., van Breugel, W., Stanford, S. A., Röttgering, H., Miley, G., \& Stern, D. 2002, AJ, 123, 637

Dey, A. 1999, in The Most Distant Radio Galaxies, ed. H. J. A. Röttgering, P. N. Best, \& M. D. Lehnert (Amsterdam: R. Netherlands Acad. Arts Sci.), 19

Dey, A., van Breugel, W., Vacca, W. D., \& Antonucci, R. 1997, ApJ, 490, 698

Dey, A., et al. 2005, ApJ, 629, 654

di Serego Alighieri, S., Cimatti, A., Fosbury, R. A. E., \& Hes, R. 1997, A\&A, 328,510

Dopita, M. A., Koratkar, A. P., Allen, M. G., Tsvetanov, A., Ford, H. C., Bicknell, G. V., \& Sutherland, R. S. 1997, ApJ, 490, 202

Dopita, M. A., \& Sutherland, R. S. 1996, ApJS, 102, 161

\section{EFERENCES}

Dunlop, J. S., Hughes, D. H., Rawlings, S., Eales, S. A., \& Ward, M. J. 1994, Nature, 370, 347

Eales, S. A., \& Rawlings, S. 1993, ApJ, 411, 67

Eisenhardt, P., \& Dickinson, M. 1992, ApJ, 399, L47

Ferrarese, L., \& Merritt, D. 2000, ApJ, 539, L9

Fosbury, R. A. E., et al. 2003, ApJ, 596, 797

Gebhardt, K., et al. 2000, ApJ, 539, L13

Graham, J. R., et al. 1994, ApJ, 420, L5

Greve, T. R., et al. 2005, MNRAS, 359, 1165

Groves, B. A., Dopita, M. A., \& Sutherland, R. S. 2004a, ApJS, 153, 9 2004b, ApJS, 153, 75

Hamann, F., \& Ferland, G. 1993, ApJ, 418, 11

Heckman, T. M. 2002, in ASP Conf. Proc. 254, Extragalactic Gas at Low Redshift, ed. J. S. Mulchaey \& J. Stocke (San Francisco: ASP), 292

Heckman, T. M., Lehnert, M. D., Strickland, D. K., \& Armus, L. 2000, ApJS, 129,493

Inskip, K. J., Best, P. N., Rawlings, S., Longair, M. S., Cotter, G., Röttgering, H. J. A., \& Eales, S. 2002a, MNRAS, 337, 1381

Inskip, K. J., Best, P. N., Röttgering, H. J. A., Rawlings, S., Cotter, G., \& Longair, M. S. 2002b, MNRAS, 337, 1407

Ivison, R. J., Dunlop, J. S., Smail, I., Dey, A., Liu, M. C., \& Graham, J. R. 2000, ApJ, 542, 27

Iwamuro, F., et al. 2003, ApJ, 598, 178

Jarvis, M. J., Wilman, R. J., Röttgering, H. J. A., \& Binette, L. 2003, MNRAS, 338,263

Kauffmann, G., \& Haehnelt, M. 2000, MNRAS, 311, 576

Kennicutt, R. C. 1998, ARA\&A, 36, 189

Lilly, S. J. 1988, ApJ, 333, 161

Loeb, A. 1993, ApJ, 403, 542

Magorrian, J., et al. 1998, AJ, 115, 2285

Martin, C. L. 1999, ApJ, 513, 156

Martin-Mirones, J. M., Martinez-Gonzalez, E., Gonzalez-Serrano, J. I., \& Sanz, J. L. 1995, ApJ, 440, 191

Massey, P., Strobel, K., Barnes, J. V., \& Anderson, E. 1988, ApJ, 328, 315

Maxfield, L., Spinrad, H., Stern, D., Dey, A., \& Dickinson, M. 2002, AJ, 123, 2321

McCarthy, P. J. 1993, ARA\&A, 31, 639

McLean, I. S., et al. 1998, Proc. SPIE, 3354, 566

Nagashima, M., Lacey, C. G., Baugh, C. M., Frenk, C. S., \& Cole, S. 2005, MNRAS, 363, 31

Neufeld, D. A. 1990, ApJ, 350, 216

Oke, J. B., et al. 1995, PASP, 107, 375

Panagia, N., Stiavelli, M., Ferguson, H., \& Stockman, H. S. 2003, Rev. Mex. AA Ser. Conf., 17, 230 
Papadopoulos, P. P., Röttgering, H. J. A., van der Werf, P. P., Guilloteau, S., Omont, A., \& van Breugel, W. J. M. 2000, ApJ, 528, 626

Pentericci, L., Van Reeven, W., Carilli, C. L., Röttgering, H. J. A., \& Miley, G. K. 2000, A\&AS, 145, 121

Pettini, M., Shapley, A. E., Steidel, C. C., Cuby, J., Dickinson, M., Moorwood, A. F. M., Adelberger, K. L., \& Giavalisco, M. 2001, ApJ, 554, 981

Rauch, M., Sargent, W. L. W., \& Barlow, T. A. 2001, ApJ, 554, 823

Reuland, M., et al. 2003, ApJ, 592, 755

Röttgering, H. J. A., van Ojik, R., Miley, G. K., Chambers, K. C., van Breugel, W. J. M., \& de Koff, S. 1997, A\&A, 326, 505

Scharf, C., Smail, I., Ivison, R., Bower, R., van Breugel, W., \& Reuland, M. 2003, ApJ, 596, 105

Sheinis, A. I., Miller, J. S., Bolte, M., \& Sutin, B. M. 2000, Proc. SPIE, 4008, 522

Silk, J., \& Rees, M. J. 1998, A\&A, 331, L1

Springel, V., Di Matteo, T., \& Hernquist, L. 2005, MNRAS, 361, 776

Steinbring, E., Crampton, D., \& Hutchings, J. B. 2002, ApJ, 569, 611

Stevens, J. A., et al. 2003, Nature, 425, 264

Tody, D. 1993, in ASP Conf. Ser. 52, Astronomical Data Analysis Software and Systems II, ed. R. J. Hanisch, R. J. V. Brissenden, \& J. Barnes (San Francisco: ASP), 173
Uson, J. M., Bagri, D. S., \& Cornwell, T. J 1991, Phys. Rev. Lett., 67, 3328 van Breugel, W., Stanford, A., Dey, A., Miley, G., Stern, D., Spinrad, H., Graham, J., \& McCarthy, P. 1999, in The Most Distant Radio Galaxies, ed. H. J. A. Röttgering, P. N. Best, \& M. D. Lehnert (Amsterdam: R. Netherlands Acad. Arts Sci.), 49

van Ojik, R., Röttgering, H. J. A., Carilli, C. L., Miley, G. K., Bremer, M. N., \& Macchetto, F. 1996, A\&A, 313, 25

van Ojik, R., Röttgering, H. J. A., Miley, G. K., \& Hunstead, R. W. 1997, A\&A, 317, 358

Vernet, J., Fosbury, R. A. E., Villar-Martín, M., Cohen, M. H., Cimatti, A., di Serego Alighieri, S., \& Goodrich, R. W. 2001, A\&A, 366, 7

Villar-Martín, M., Fosbury, R. A. E., Binette, L., Tadhunter, C. N., \& RoccaVolmerange, B. 1999, A\&A, 351, 47

Villar-Martín, M., Vernet, J., di Serego Alighieri, S., Fosbury, R., Humphrey, A., \& Pentericci, L. 2003, MNRAS, 346, 273

Wilman, R. J., Gerssen, J., Bower, R. G., Morris, S. L., Bacon, R., de Zeeuw, P. T., \& Davies, R. L. 2005, Nature, 436, 227

Wilman, R. J., Jarvis, M. J., Röttgering, H. J. A., \& Binette, L. 2004, MNRAS, 351,1109 